\title{
Drift on holey landscapes as a dominant evolutionary process
}

Ned A. Dochtermann a,b Brady Klock ${ }^{a,}$, , Derek A. Roff c, ${ }^{\text {, }}$ \& Raphaël Royauté d, ${ }^{*}$

a Department of Biological Sciences; North Dakota State University

b ned.dochtermann@gmail.com

c Department of Biology; University of California, Riverside

d Senckenberg Biodiversity and Climate Research Centre, Frankfurt, Germany

* these authors are listed in alphabetical order

Phenotypes typically display integration, i.e. correlations between traits. For quantitative traits - like many behaviors, physiological processes, and life-history traits-patterns of integration are often assumed to have been shaped by the combination of linear, non-linear, and correlated selection, with trait correlations representative of optimal combinations. Unfortunately, this assumption has rarely been critically tested, in part due to a lack of clear alternatives. Here we show that trait integration across 6 phyla and 60 species (including both Plantae and Animalia) is consistent with evolution across high dimensional "holey landscapes" rather than classical models of selection. This suggests that the leading conceptualizations and modeling of the evolution of trait integration fail to capture how phenotypes are shaped. Instead, traits are integrated in a manner contrary to predictions of dominant evolutionary theory.

1 A common attribute of most organisms is that they display trait integration. For example,

2 life-history traits are often correlated according to a slow-fast continuum ${ }^{1,2}$. This trait

3 integration is commonly understood in terms of trade-offs and fitness maximization ${ }^{3-8}$ and

4 is frequently modeled as populations moving across adaptive landscapes toward peaks of

5 higher fitness. However, this adaptive perspective has rarely been evaluated due to a lack

6 of clear alternatives. Consequently, much of our understanding of when and why

7 quantitative traits are correlated might be shaped by adaptive just-so-stories 9 .

\section{Competing evolutionary processes}

8 Our understanding of selection has been strongly shaped by Sewall Wright's

9 conceptualization of an adaptive landscape, with populations moving from areas of low 
10 fitness to areas of higher fitness 10,11 . While

11 the simple one and two trait landscapes

12 Wright originally detailed have been

13 criticized as unrealistic, including by

14 Wright himself 10 , the general metaphor has

15 nonetheless guided much of evolutionary

16 thought ${ }^{12}$.

For quantitative traits, like many aspects of physiology, behavior, and morphology, Wright's metaphor has been mathematically extended to complex topographies with ridges or tunnels of high fitness 13,14 15. Applying these adaptive landscape topologies in mathematical models has led to insights into how variation in traits, and correlations among traits, are expected to change over time ${ }^{15}$. Simulations have similarly led to the prediction that landscapes with complex topographic features like fitness ridges result in populations with genetic correlations aligned with these ridges ${ }^{3-5}$.

\section{Concurrent to the study of} quantitative trait variation, the question of 4 how the topography of fitness landscapes 5 affects sequence evolution at the genomic level has garnered similar interest 16.

\section{Whereas theoreticians interested in} quantitative trait variation have focused on

9 relatively simple landscapes e.g. 3,4,5,17-19,

40 theoretical research regarding sequence

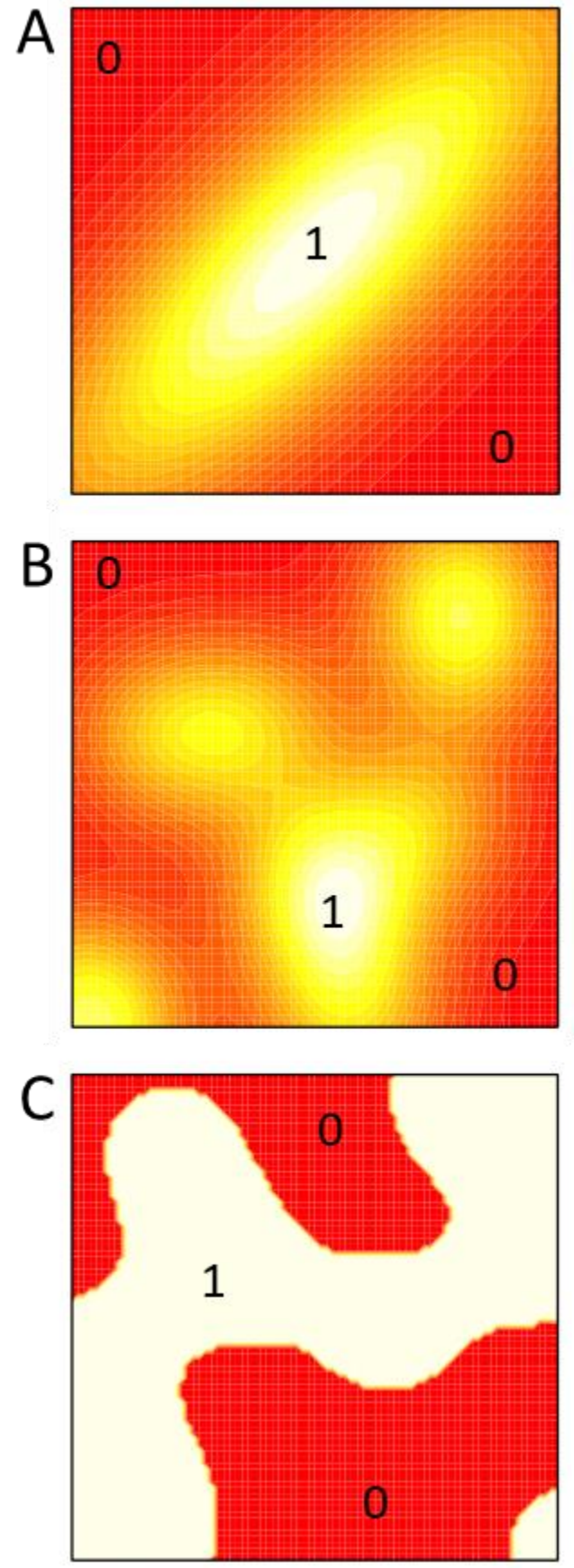

Figure 1. Example fitness landscapes. Hotter colors correspond to higher fitness. A. A simple single peak Fujiyama landscape with a single optimum (1). B. A more rugged landscape with multiple local optima and a single global optimum. C. A simplified Holey landscape where particular combinations of values correspond to high, average, fitness (1) or low (0) fitness. 
41 evolution has spanned simple single peak Gaussian "Fujiyama landscapes", to "badlands

42 landscapes" Fig 1A \& 1B; ${ }^{20}$, to abstract high-dimensional "holey landscapes" Fig 1C; ${ }^{21}$.

43 Among other topics, this research has explored how topographies of varying complexity

44 (Fig 1) affect the ability of populations to reach optima ${ }^{16}$. An important conclusion from

45 this research is that evolutionary dynamics on simple landscapes often fail to properly

46 predict evolution on landscapes of higher dimensionality.

Of these landscapes, perhaps most conceptually unfamiliar and unintuitive to researchers focused on quantitative trait evolution are Gavrilets' (1997) holey landscapes

(Fig 1C). The general concept of holey landscapes is that, because phenotypes are made up of a large number of traits, phenotypes are necessarily high dimensional constructs and corresponding landscapes will consist of either trait combinations that are of average fitness or trait combinations that confer low fitness or are inviable 21,22 . This results in flat landscapes with holes at inviable or low fitness phenotypes (Fig 1C). The flat landscape can be understood as stemming from the full multivariate nature of the phenotype: while there may be clear fitness differences in two dimensions, strong gradients will create holes in the landscape and peaks will average out when additional traits are considered. Unfortunately, predictions about quantitative trait evolution on holey landscapes are not clear.

More broadly, it is not clear what the topography of landscapes typically is for natural populations. While portions of selection surfaces and fitness landscapes can be directly estimated 23,24 , these estimates may differ from the underlying full landscape due to several factors. These include: the omission of fitness affecting traits ${ }^{25}$, incomplete estimation of fitness 26,27 , and insufficient power to estimate non-linear selection coefficients ${ }^{28}$. An alternative to direct estimation of adaptive landscape topography is to infer landscape topography from observed trait (co)variances. For example, low additive genetic variation is suggestive of stabilizing or directional selection ${ }^{29}$ and additive genetic correlations are expected to emerge from correlational selection and fitness ridges in a landscape e.g. ${ }^{13,14}$. Thus, an ability to gain an understanding of the topography of adaptive

68 landscapes based on observed trait variation would aid our understanding as to how selection is realized in natural populations.

Here we used a simulation model to examine how evolution on different landscapes 
72 drift, that evolved via adaptation on simple Gaussian fitness landscapes stemming from

73 Wright's metaphor, or that evolved on holey landscapes. This allowed us to generate

74 testable predictions for how the structure of additive genetic variances and covariances (G)

75 are shaped by different landscape topographies. We next compared these modeled

76 outcomes to 181 estimates of $\mathbf{G}$, representing 60 species from 6 phyla, including both

77 plants and animals, to determine if observed trait integration is consistent with any of the

78 modeled processes.

Model Construction

80 We developed an individual variance components model (Methods, Fig S1; ${ }^{30}$ ) wherein

81 individuals had phenotypes comprised of 10 traits $(k)$, with each trait being highly

82 heritable $\left(\mathrm{h}^{2}=0.8\right)$, and initial genetic covariances between traits set at zero. Populations

83 of individuals evolved on one of five landscapes: (i) a flat landscape where no selection

84 occurred (i.e. drift alone), (ii) Gaussian landscapes where fitness for each pair of traits was

85 characterized by a single peak but with correlational selection, and three (iii - v)

86 implementations of holey landscapes differing by $p^{21,22}$, the proportion of viable

87 phenotypes in a holey landscape $(p=0.2,0.5$, and 0.8$)$. Each of the modeling scenarios was

88 simulated 250 times for populations of 7500 individuals and for 100 generations for each

89 population. Full modeling details are provided in the Methods and all modeling code is

90 available at https://github.com/DochtermannLab/Wright vs Holey.

\section{$91 \quad$ Model analysis}

Following these simulations, the eigen structures of the resulting 1250 population genetic covariance matrices were compared. Because the simulated phenotypes consisted

94 of 10 traits, it was the overall multivariate pattern of variation that was of interest rather 95 than any specific single trait or pairwise combination. To do so, we calculated the ratio of 96 each matrix's second eigen value $\left(\lambda_{2}\right)$ to its dominant eigen value (i.e. $\left.\lambda_{2} / \lambda_{1}\right)$. This metric

97 provides a better estimate of the compression of variance into a leading dimension than do 98 other common metrics like the variation of the first eigen value to the sum of eigen values 99 (i.e. $\lambda_{1} / \sum \lambda$ ). For example, $\lambda_{1} / \sum \lambda$ could be low if the variation not captured by $\lambda_{1}$ is equally 
distributed across all other dimensions, even if all other dimensions contained relatively little variation. The same scenario would produce a high value for $\lambda_{2} / \lambda_{1}$. and Tukey post-hoc testing. Alternative metrics for characterizing covariance matrices were consistent with the results for $\lambda_{2} / \lambda_{1}$ (see Supplementary Results). We also present the results of analyses of a broad range of starting conditions and model conditions in the

106 Supplementary Results. These supplemental analyses confirmed the robustness of the 107 findings reported below.

Model outcomes

109 When evolving on holey landscapes, populations lost greater relative variation in the non110 dominant dimensions as compared to when evolving on simple Gaussian landscapes or 111 when subject solely to drift (Fig 2; Fig S3 A-D). $\lambda_{2} / \lambda_{1}$ significantly differed depending on 112 selection regime $\left(\mathrm{F}_{4,1245}=368, \mathrm{p}<0.01\right.$; Fig 2$)$. Populations experiencing either just drift 113 or evolving on Gaussian landscapes maintained a more even amount of variation across 114 dimensions compared to those evolving on holey landscapes (i.e. higher $\lambda_{2} / \lambda_{1}$ all post-hoc 115 comparisons $\mathrm{p}<0.001$; Fig 2, Table S3). All populations evolving on holey landscapes 116 exhibited similar $\lambda_{2} / \lambda_{1}$ ratios regardless of $p$ (all post-hoc comparisons of outcomes for 117 holey landscapes: $\mathrm{p}>0.05$; Fig 2, Table S3). While a modest difference, populations 118 evolving due to drift alone also exhibited a significantly greater ratio than populations 119 evolving on Gaussian landscapes (difference $=0.06, \mathrm{p}=0.002$; Fig 2, Table S3). This 120 magnitude of a difference is unlikely to be biologically important or detectable in natural 121 populations and instead is likely driven by the high power available with simulations.

122 These differences were consistent across approaches to summarizing $\mathbf{G}$ and are robust to 123 conditions of the simulations (Supplementary Results).

$124 \quad$ These modeling results generate the general prediction that greater relative variation 125 in multiple dimensions is maintained when populations evolve on Gaussian landscapes than 126 when evolving on holey landscapes. Put another way, evolving on holey landscapes is 127 predicted to result in a large decrease in variation from the dominant to subsequent 128 dimensions and, consequently, a lower $\lambda_{2} / \lambda_{1}$ value (Fig S3). 


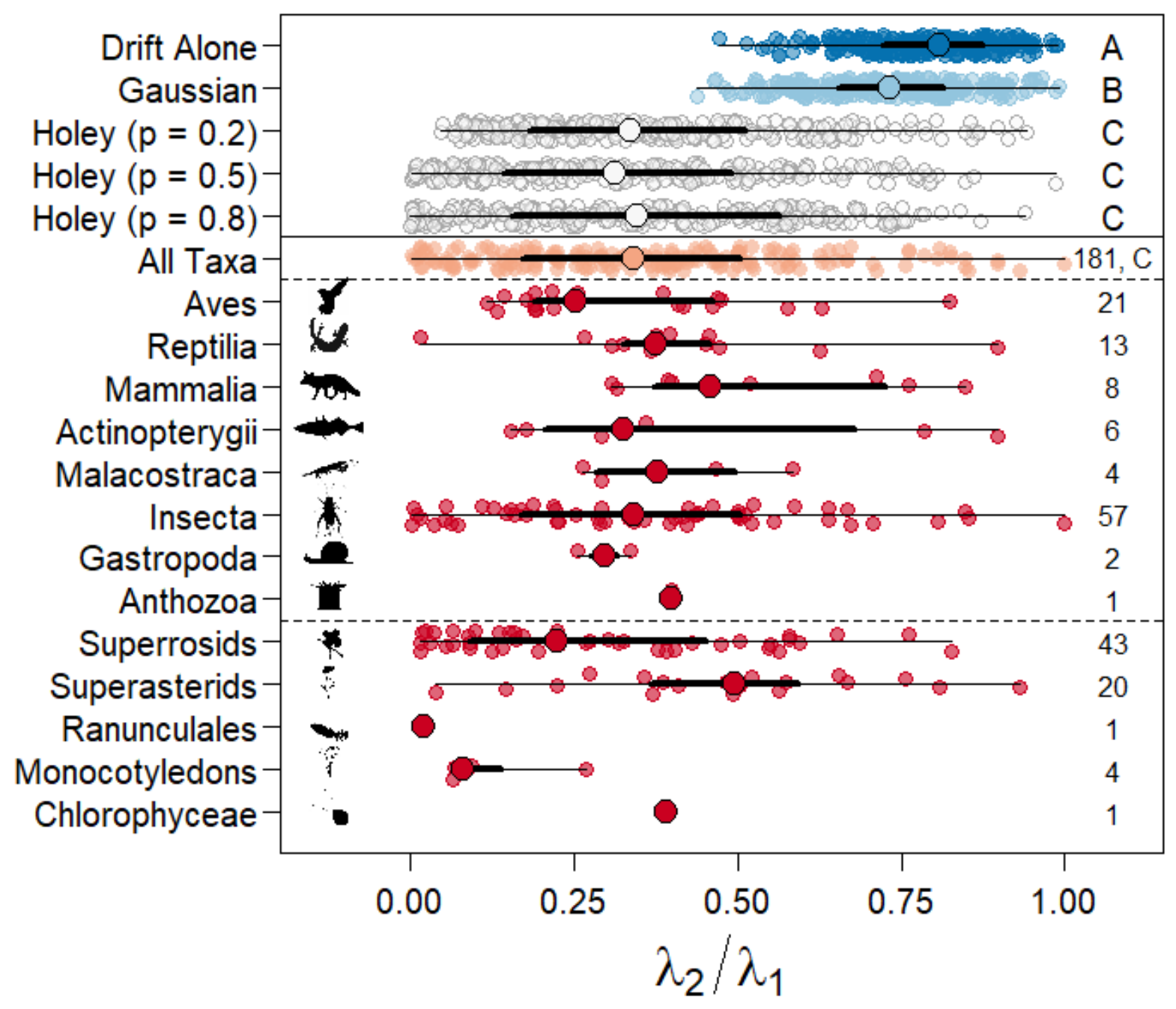

Figure 2. Modified "Orchard plot" of $\lambda_{2} / \lambda_{1}$ values for simulated (above solid line) and observed G matrices. Trunks (large points) are the medians for the specified group (e.g. Gaussian landscapes or Insecta), branches (thick lines) are interquartile ranges, twigs (thin lines) give the full range of values, and fruits (smaller points) are individual estimates within a simulation or taxonomic group. Rightmost letters correspond to statistical significance-or lack thereof-of comparisons of ratios among simulations. Datasets sharing letters did not significantly differ (Table S3). Populations evolving due to drift alone had a significantly higher ratio than observed for either stabilizing selection or evolution on any of the holey landscapes. Populations evolving on holey landscapes also had lower ratios than those experiencing stabilizing selection but did not differ from each other. Rightmost numbers are the number of estimates available via literature search. (organism silhouettes courtesy of phylopic.org, Public Domain Mark 1 licenses or CCA 3.0; Chlorophyceae: S.A. Muñoz-Gómez, Superrosid: D.J. Bruzzese, Superasterid: T.M. Keesey \& Nadiatalent).

\section{Observed patterns of trait integration}

131 We next sought to determine which of the modeled processes produced results consistent 132 with observed patterns of trait integration. To do so we conducted a literature review 
133 wherein we used Web of Science to search the journals American Naturalist, Ecology and

134 Evolution, Evolution, Evolutionary Applications, Evolutionary Ecology, Genetics, Heredity,

135 Journal of Evolutionary Biology, Journal of Heredity, Nature Ecology and Evolution, and the

136 Proceedings of the Royal Society (B). We searched these journals using the terms "G

137 matrix" on 14 May 2019, yielding a total of 272 articles. Each article was reviewed and

138 estimated $\mathbf{G}$ matrices extracted if the article met inclusion criteria. For inclusion, an

139 estimated $\mathbf{G}$ matrix must have been estimated for more than 2 traits (i.e. $>2 \times 2$ ), must

140 have been reported as variances and covariances (i.e. not genetic correlations), and must

141 not have been estimated for humans. Based on these inclusion criteria, we ended up with a

142 dataset of 181 estimated $\mathbf{G}$ matrices from 60 articles (Fig S2). For each published G matrix,

143 we estimated $\lambda_{2} / \lambda_{1}$.

\section{Observed outcomes}

145 Across all taxa, average $\lambda_{2} / \lambda_{1}$ was 0.36 (sd: 0.23 , Fig 2). This estimate is consistent and

146 statistically indistinguishable from those observed for simulated populations evolving on

147 Holey landscapes ( $\mathrm{t}_{\mathrm{df}} 17.275=0.32,1.20,-0.05, \mathrm{p}>0.2$ (all) versus Holey landscapes with $p=$

$148 \quad 0.2,0.5$, and 0.8 respectively; Fig 2, Table S10) and substantially less than observed for

149 simulated populations that evolved on Gaussian landscapes or via drift alone $\left(t_{d f: 17.275}=-\right.$

$15012.42,-14.55$ respectively, $\mathrm{p}<0.001$ (both)).

151 While some individual estimates at the species level exhibited high $\lambda_{2} / \lambda_{1}$ values (Fig

152 2), phylogeny explained little variation in these values (phylogenetic heritability $=0.05$;

153 Table S9). As was the case across all taxa, median $\lambda_{2} / \lambda_{1}$ values for each taxonomic Class (or

154 comparable level clade) were consistently lower than expected if evolution occurred on

155 Gaussian landscapes or via drift alone (Fig 2). Instead, these results are strongly consistent

156 with evolution on Holey landscapes.

\section{Conclusions}

158 The observation that traits linked to fitness are frequently correlated has been a major

159 driver of research across evolutionary ecology. Research in life-history, physiology, and

160 behavior has frequently been structured around such observations, arguing that this

161 integration stems from optimization in the face of trade-offs 1,2,31-33. However, because 
162 selection is frequently acting on many traits, patterns of integration quickly diverge from

163 simple expectations, even under conventional models of evolution. However, our results

164 suggest something substantively different is occurring: the observed pattern of variation

165 across taxa suggests that classic models of the evolution of quantitative traits-e.g. stabilizing and correlational selection-are not what have predominantly shaped trait integration.

167 Instead, drift across holey landscapes ${ }^{21,22}$ is more consistent with observed quantitative 168 genetic variation (Fig 2).

169 Much of the early theoretical development of holey landscapes focused on the ability

170 of populations to traverse genomic sequence differences via drift, with some sequences

171 being inviable (e.g. due to missense differences in coding regions). How this extends to

172 quantitative traits had been less clear. Our simulation model provides one approach to

173 applying the holey landscape framework to quantitative traits, treating each trait as a

174 threshold character ${ }^{34}$. Other approaches to modeling quantitative traits on holey

175 landscapes and evolution in response to these versions, such as the generalized Russian

176 roulette model 22 , may produce different outcomes. It is also important to recognize that

177 the broad support for evolution on holey landscapes does not preclude that subsets of

178 traits from having evolved on Gaussian landscapes. Indeed, stabilizing selection has been

179 observed in natural populations ${ }^{28}$, though understanding its general strength even on a

180 case by case basis is confounded with methodological problems ${ }^{35,36}$. Regardless, our

181 finding that observed patterns of quantitative genetic variation across taxonomic groups

182 are not consistent with traditional evolutionary models stands.

183 This disconnect between observed patterns of multivariate variation and

184 expectations under conventional models of selection suggests that Wright's metaphor of

185 fitness landscapes and the subsequent implementation of this metaphor as Gaussian

186 surfaces may have contributed to an improper, or at least incomplete, understanding of

187 how selection has shaped phenotypes. A potential contributor to this problem has been the

188 lack of clear alternative explanations besides a simple null hypothesis of drift with no

189 selection. Moving forward, clear development of alternative models of the action of

190 selection and evolution in multivariate space are needed. 


\section{Acknowledgements}

The authors thank A.J. Wilson and B. de Bivort for helpful conversations. This work was supported by US NSF IOS grant 1557951 to N.A.D.

\section{Author Contributions}

NAD conceived of the project and developed the first version of the model. BK collected published $\mathbf{G}$ matrices and calculated matrix summary estimates. RR contributed to model development and analyses. DAR contributed to model development and developed the parameter exploration scheme. All authors contributed to the writing of the manuscript.

1 Wright, J., Solbu, E. B. \& Engen, S. Contrasting patterns of density-dependent selection at different life stages can create more than one fast-slow axis of life-history variation. Ecol. Evol. 10, 3068-3078 (2020).

2 Ricklefs, R. E. \& Wikelski, M. The physiology/life-history nexus. Trends in Ecology \& Evolution 17, 462-468 (2002).

3 Arnold, S. J., Burger, R., Hohenlohe, P. A., Ajie, B. C. \& Jones, A. G. Understanding the evolution and stability of the G-matrix. Evolution 62, 2451-2461, doi:10.1111/j.15585646.2008.00472.x (2008).

4 Jones, A. G., Arnold, S. J. \& Borger, R. Stability of the G-matrix in a population experiencing pleiotropic mutation, stabilizing selection, and genetic drift. Evolution 57, 1747-1760 (2003).

5 Jones, A. G., Arnold, S. J. \& Burger, R. Evolution and stability of the G-matrix on a landscape with a moving optimum. Evolution 58, 1639-1654 (2004).

6 Roff, D. A. Life history evolution. (Sinauer Associates, Inc., 2002).

7 Van Noordwijk, A. J. \& de Jong, G. Acquisition and allocation of resources: their influence on variation in life history tactics. American Naturalist, 137-142 (1986). Critique Of The Adaptationist Program. Proceedings Of The Royal Society Of London Series BBiological Sciences 205, 581-598 (1979).

10 Wright, S. Surfaces of selective value revisited. The American Naturalist 131, 115-123 (1988).

11 Wright, S. The roles of mutation, inbreeding, crossbreeding and selection in evolution, pp. 356-366 in Proceedings of the Sixth International Congress of Genetics, edited by D. Jones. Ithaca, $N Y$ (1932).

12 Olson, M. E., Arroyo-Santos, A. \& Vergara-Silva, F. A user's guide to metaphors in ecology and evolution. Trends in ecology \& evolution (2019).

13 Armbruster, W. \& Schwaegerle, K. Causes of covariation of phenotypic traits among populations. Journal of Evolutionary Biology 9, 261-276 (1996). and pollination of Dalechampia blossoms. The American Naturalist 135, 14-31 (1990). 
15 Phillips, P. C. \& Arnold, S. J. Visualizing multivariate selection. Evolution 43, 1209-1222 (1989).

16 Kauffman, S. \& Levin, S. Towards a general theory of adaptive walks on rugged landscapes. Journal of Theoretical Biology 128, 11-45 (1987).

17 Bulmer, M. The genetic variability of polygenic characters under optimizing selection, mutation and drift. Genetics Research 19, 17-25 (1972).

18 Reeve, J. P. Predicting long-term response to selection. Genetics Research 75, 83-94 (2000).

19 Turelli, M. Phenotypic evolution, constant covariances, and the maintenance of additive variance. Evolution 42, 1342-1347 (1988).

20 Kauffman, S. A. \& Weinberger, E. D. The NK model of rugged fitness landscapes and its application to maturation of the immune response. Journal of theoretical biology 141, 211245 (1989).

21 Gavrilets, S. Evolution and speciation on holey adaptive landscapes. Trends in ecology \& evolution 12, 307-312 (1997).

22 Gavrilets, S. Fitness Landscapes and the Origin of Species. (Princeton University Press, 2004).

23 Lande, R. \& Arnold, S. J. The measurement of selection on correlated characters. Evolution 37, 1210-1226 (1983).

24 Morrissey, M. B. In search of the best methods for multivariate selection analysis. Methods Ecol. Evol. 5, 1095-1109 (2014).

25 Delcourt, M., Blows, M. W., Aguirre, J. D. \& Rundle, H. D. Evolutionary optimum for male sexual traits characterized using the multivariate Robertson-Price Identity. Proceedings of the National Academy of Sciences 109, 10414-10419 (2012).

26 Arnold, S. J. \& Wade, M. J. On the measurement of natural and sexual selection: theory. Evolution 38, 709-719 (1984).

27 Shaw, R. G. \& Geyer, C. J. Inferring fitness landscapes. Evolution 64, 2510-2520 (2010).

28 Kingsolver, J. G. et al. The strength of phenotypic selection in natural populations. The American Naturalist 157, 245-261 (2001).

29 Mousseau, T. A. \& Roff, D. A. Natural selection and the heritability of fitness components. Heredity 59, 181-197 (1987).

30 Roff, D. Modeling evolution: an introduction to numerical methods. (Oxford University Press, 2010).

31 Roff, D. A. \& Fairbairn, D. J. The evolution of trade-offs: where are we? Journal of Evolutionary Biology 20, 433-447 (2007).

32 Réale, D. et al. Personality and the emergence of the pace-of-life syndrome concept at the population level. Philos. Trans. R. Soc. B-Biol. Sci. 365, 4051-4063, doi:10.1098/rstb.2010.0208 (2010).

33 Houle, D. Genetic covariance of fitness correlates--what genetic correlations are made of and why it matters. Evolution 45, 630-648, doi:10.1111/j.1558-5646.1991.tb04334.x (1991).

34 Lynch, M. \& Walsh, B. Genetics and Analysis of Quantitative Traits. 980 (Sinauer Associates, 1998).

35 Morrissey, M. B. Meta-analysis of magnitudes, differences and variation in evolutionary parameters. Journal of Evolutionary Biology 29, 1882-1904 (2016).

36 Stinchcombe, J. R., Agrawal, A. F., Hohenlohe, P. A., Arnold, S. J. \& Blows, M. W. Estimating nonlinear selection gradients using quadratic regression coefficients: double or nothing? Evolution: International Journal of Organic Evolution 62, 2435-2440 (2008).

37 Lewandowski, D., Kurowicka, D. \& Joe, H. Generating random correlation matrices based on vines and extended onion method. Journal of multivariate analysis 100, 1989-2001 (2009).

38 Lande, R. Genetic variation and phenotypic evolution during allopatric speciation. The American Naturalist 116, 463-479 (1980). 
39 Hansen, T. F. \& Houle, D. Measuring and comparing evolvability and constraint in multivariate characters. Journal of Evolutionary Biology 21, 1201-1219, doi:10.1111/j.1420-

40 Kirkpatrick, M. Patterns of quantitative genetic variation in multiple dimensions. Genetica

41 Nakagawa, S. \& Santos, E. S. Methodological issues and advances in biological meta-analysis. Evolutionary Ecology 26, 1253-1274 (2012).

42 Bates, D., Mächler, M., Bolker, B. \& Walker, S. Fitting linear mixed-effects models using lme4. arXiv preprint arXiv:1406.5823 (2014). (2008). 


\section{Supplemental Methods}

Simulation Models

\section{Model Construction}

290 We developed an individual variance components model Fig S1; sensu ${ }^{30}$ wherein

291 individuals had phenotypes comprised of 10 traits $(k)$ and with each trait being highly

292 heritable $\left(h^{2}=0.8\right)$ and initial genetic covariances between traits of 0 . A high heritability

293 was initially used to reduce the number of generations needed to determine the response

294 of populations to selection. Genetic covariances were set to an initial value of zero to

295 simulate a population under linkage equilibrium. Viability selection was applied based on

296 fitness, which was determined either by location on a ten-dimensional holey landscape or

297 on simple Gaussian landscapes with a single optimum per trait pair.

\section{Holey Landscapes}

For simulations evaluating holey landscapes, we simulated populations in which

300 traits were inherited as though continuous but expressed categorically as one of two

301 phenotypic variants (e.g. phenotype 0 versus 1 for trait 1 ). Specifically, at the start of

302 simulations, we drew genotypes for each individual from a normal distribution with a

303 mean of zero and standard deviation of 1 . To these normally distributed genotypes, we

304 added "environmental" values $(\mu=0$, all covariances $=0)$ to generate a phenotype with a

305 heritability of 0.8 . These continuously distributed phenotypic values were then

306 transformed as one implementation of the holey landscape is based on the fitness of

307 specific and discrete combinations. Specifically, the continuously distributed values were

308 transformed to be a phenotype of 0 or 1 , with a genotype $<0$ being " 0 " and a genotype $>0$

309 being “1” (Table S1). 
The holey landscape for a specific simulation was then constructed by randomly

311 assigning a fitness of 0 or 1 to the 1024 possible phenotypes $\left(2^{k}\right)$ trait combinations based

312 on the parameter $p$. " $p$ " was the probability that a trait combination had a fitness of 1 and

313 corresponds to Gavrilets' (2004) percolation parameter. We used three values of $p$ in our

314 simulation ranging from weak $(p=0.2)$, moderate $(p=0.5)$ and high $(p=0.8) \cdot p$ can vary

315 between 0 and 1 , with values of 1 corresponding to a landscape where all trait

316 combinations are viable and have a fitness of 1 . As $p$ approaches 0 , few trait combinations

317 are viable.

318 After the first generation, genotypes were drawn from a multivariate normal

319 distribution based on the means and genetic variance-covariance matrix of the population

320 that survived selection. Environmental contributions again had an average of 0 and no

321 environmental correlation with a variance set to keep heritability at 0.8 (or other values

322 during parameter exploration, below). The resulting phenotypic values were then

323 converted to 0's and 1's as above. This approach to generating subsequent generations

324 follows the structure of individual variance components models described by Roff ${ }^{30}$. We

325 used this individual variance components approach rather than an agent-based approach

326 as the latter combined with the computational requirements of matching phenotypes to

327 fitness under the holey landscape model was not amenable to simulation analysis. 
Table S1. Example conversion of an underlying genotype to a phenotype under the two modelling scenarios. The same individual has a genotypic value for each of the 10 traits simulated (e.g. - 0.918 for trait 10). To this, "environmental" contributions are added, taking heritability to 0.8. For Holey Landscape simulations, these phenotypic values are then converted to either 0 or 1 based on whether the phenotype is negative or positive.

\begin{tabular}{lcccccccccc}
\hline & \multicolumn{10}{c}{ Trait } \\
\hline & 1 & 2 & 3 & 4 & 5 & 6 & 7 & 8 & 9 & 10 \\
\hline Genotype & 0.008 & 0.770 & 0.477 & 0.112 & -0.512 & 0.751 & -1.752 & -0.944 & 0.030 & -0.918 \\
\hline $\begin{array}{l}\text { Environmental } \\
\text { Contribution }\end{array}$ & 0.402 & -0.221 & 0.023 & 0.053 & 0.082 & -0.25 & 0.63 & 0.285 & -0.007 & 0.271 \\
\hline $\begin{array}{l}\text { Holey Landscape } \\
\text { Phenotype }\end{array}$ & 1 & 1 & 1 & 1 & 0 & 1 & 0 & 0 & 1 & 0 \\
\hline $\begin{array}{l}\text { Gaussian } \\
\begin{array}{l}\text { Landscape } \\
\text { Phenotype }\end{array}\end{array}$ & 0.410 & 0.549 & 0.500 & 0.165 & -0.430 & 0.501 & -1.122 & -0.659 & 0.023 & -0.647 \\
\hline
\end{tabular}

334 the landscape for each pair of traits (e.g. $\omega_{i, j}$ ) was defined as $\left[\begin{array}{cc}1 & \omega_{i, j} \\ \omega_{i, j} & 1\end{array}\right]$ consistent with

335 previous simulation studies examining the evolution of quantitative traits reviewed by ${ }^{3}$.

336 This approach corresponds to single peak landscapes in any two dimensions. The forty-five

$337 \omega_{i, j}$ values that fully describe the landscape were generated using the LKJ onion method for

338 constructing random correlation matrices with a flat distribution of correlations $(\eta=1$;

339 Lewandowski et al. 2009). Using the LKJ onion method ensures that the full description of

340 the landscape $(\omega)$ is positive semi-definite with feasible partial correlations. We then

341 calculated each individual's fitness based on a Gaussian surface ${ }^{38}$ : 


$$
w_{h}=\exp \left(-.5\left(\mathrm{z}_{h}-\theta\right)^{\mathrm{T}} \omega^{-1}\left(\mathrm{zh}^{-}-\theta\right)\right)
$$

343 where $w h$ is the fitness of individual $h, z h$ is a vector of the observed phenotypic values for

344 individual $h, \omega$ is the selection surface, and $\theta$ is the optima for traits $(0)$. Truncation

345 selection was applied based on fitness, with the $50 \%$ of individuals possessing the highest

346 fitness surviving (main results). In an additional set of simulations, stronger truncation

347 selection was applied and only $10 \%$ of the population survived.

$348 \quad$ Following selection in either framework, the next generation was constructed using

349 an individual variance components approach ${ }^{30}$. Specifically, the next generation was

350 generated as described above based on the trait means, variances and covariances of

351 survivors. Selection therefore acted via changes in means and variances and drift during

352 the selection simulations was due to sampling error from the selection shaped phenotypic

353 distributions.

354 Drift alone

355 For populations evolving via drift alone phenotypes were generated as for Gaussian

356 adaptive landscapes. Composition of subsequent generations was likewise generated based

357 on the means and variances of the prior generation, without selection. The drift model

358 therefore was simply a model of sampling error.

359 Each of five modeling scenarios (simple landscapes, drift alone, three Holey

360 landscapes with $p=0.2,0.5$, or 0.8 ) was simulated 250 times for populations of 7500

361 individuals and for 100 generations for each population. All modeling code is available at

362 https://github.com/DochtermannLab/Wright vs Holey.

363 Statistical Comparison of Evolutionary Metrics 
364 To clarify differences in evolutionary outcomes across modeling scenarios, we

365 summarized evolutionary outcomes at the level of $\mathbf{G}$ matrices based on several metrics:

366 1. $\lambda_{2} / \lambda_{1}$; results for this metric are presented in the main text

367 2. $\lambda_{1} / \sum \lambda$; this is a commonly used summary value and represents the proportion of variation captured by dominant eigenvalue. This can be interpreted as the proportion variation in the main dimension of covariance to whether a particular process results in the loss of more or less variation multivariate space how constrained evolutionary responses are based on correlations. At the extreme, an average autonomy of 0 would indicate absolute constraints on responses to selection and an average autonomy of 1 indicates evolutionary independence. Values between 0 and 1 represent quantitative constraints.

380 following the main text, based on ANOVA followed by post-hoc comparisons based on 381 calculation of Tukey's Honest Significant Differences (HSD).

383 The above modeling scenarios were used for our overall general analyses and for

384 comparison to observed values. However, to explore whether our modeling outcomes were 385 due to fundamentally different and generalizable outcomes or instead emerged from 386 peculiarities of initial parameters, we expanded our analyses in two ways. 
First, in addition to the moderate/weak strength of truncation selection modeled

388 above (0.5), we also modeled stronger selection where only $10 \%$ of individuals survived.

389 For this stronger strength of selection we again conducted 250 simulations of 7500

390 individuals for 100 generations. These simulations were included in the above analyses.

391 Second, to more broadly examine the sensitivity of our results to different starting

392 values, we conducted simulation studies for our selection model, our model of drift, and

393 our model of evolution on flat holey landscapes. For each modeling scenario (Gaussian

394 surfaces, drift, Holey landscapes) we conducted 1000 simulations where both the

395 magnitude of initial genetic variation in each trait varied and $h^{2}$ varied $\left(h^{2}\right.$ was defined

396 independently). For each scenario we then explored how other changes in starting

397 parameters affected the eigenstructure of G (Table S2).

399 within modeling scenario-using linear models. All two-way interactions were included in

400 analyses and variables (model parameters) were mean centered but unscaled. We then

401 qualitatively compared $\lambda_{2} / \lambda_{1}$ across modeling scenarios based on heat plots. 
Table S2. Parameters varied across simulation iterations by modeling scenario and range of possible values

\begin{tabular}{|c|c|c|}
\hline Modeling Scenario & Parameter varied & Parameter values \\
\hline \multirow{4}{*}{ Gaussian surfaces } & $\begin{array}{l}\text { Genetic variation } \\
\text { present in traits }\end{array}$ & $\begin{array}{l}\text { Single trait variabilities were independently drawn from } \\
\text { uniform distributions ranging from } 0.1 \text { to } 1.9 \text {. }\end{array}$ \\
\hline & $\begin{array}{l}\text { Correlations among } \\
\text { traits }\end{array}$ & $\begin{array}{l}\text { Initial genetic correlations were drawn according to the } \\
\text { LKJ onion method }{ }^{37} \text { with } \eta=1 \text {. }\end{array}$ \\
\hline & $\mathrm{h}^{2}$ & $\begin{array}{l}\text { Heritabilities were drawn from a uniform distribution } \\
\text { ranging from } 0.01 \text { to } 0.99\end{array}$ \\
\hline & Selection strength & $\begin{array}{l}\text { Proportion of individuals surviving to reproduce was } \\
\text { drawn from a uniform distribution ranging from } 0.1 \text { to } 0.9 \text {. }\end{array}$ \\
\hline \multirow{3}{*}{ Drift } & $\begin{array}{l}\text { Genetic variation } \\
\text { present in traits }\end{array}$ & $\begin{array}{l}\text { Single trait variabilities were independently drawn from } \\
\text { uniform distributions ranging from } 0.1 \text { to } 1.9 \text {. }\end{array}$ \\
\hline & $\begin{array}{l}\text { Correlations among } \\
\text { traits }\end{array}$ & $\begin{array}{l}\text { Initial genetic correlations were drawn according to the } \\
\text { LKJ onion method }{ }^{37} \text { with } \eta=1 \text {. }\end{array}$ \\
\hline & $\mathrm{h}^{2}$ & $\begin{array}{l}\text { Heritabilities were drawn from a uniform distribution } \\
\text { ranging from } 0.01 \text { to } 0.99\end{array}$ \\
\hline \multirow{4}{*}{ Holey landscapes } & $\begin{array}{l}\text { Genetic variation } \\
\text { present in traits }\end{array}$ & $\begin{array}{l}\text { Single trait variabilities were independently drawn from } \\
\text { uniform distributions ranging from } 0.1 \text { to } 1.9 \text {. }\end{array}$ \\
\hline & $\begin{array}{l}\text { Correlations among } \\
\text { traits }\end{array}$ & $\begin{array}{l}\text { Initial genetic correlations were drawn according to the } \\
\text { LKJ onion method }{ }^{37} \text { with } \eta=1 \text {. }\end{array}$ \\
\hline & $\mathrm{h}^{2}$ & $\begin{array}{l}\text { Heritabilities were drawn from a uniform distribution } \\
\text { ranging from } 0.01 \text { to } 0.99\end{array}$ \\
\hline & $p$ & $\begin{array}{l}\text { Proportion of inviable phenotypes, Gavrilets' percolation } \\
\text { parameter }\end{array}$ \\
\hline
\end{tabular}

$404 \quad$ Empirically Estimated G Matrices

405 Observed patterns of multivariate genetic variation

406 We conducted a literature review with Web of Science to search the journals American

407 Naturalist, Ecology and Evolution, Evolution, Evolutionary Applications, Evolutionary

408 Ecology, Genetics, Heredity, Journal of Evolutionary Biology, Journal of Heredity, Nature

409 Ecology and Evolution, and the Proceedings of the Royal Society (B). These journals were

410 searched using the terms "G matrix" on 14 May 2019, yielding a total of 272 articles. Each

411 article was reviewed to determine if the article met inclusion criteria. Our inclusion criteria

412 were:

413 1. A G matrix must have been estimated for more than 2 traits (i.e. $>2 \times 2$ )

4142 2. Must have been reported as variances and covariances (i.e. not genetic correlations)

4153 . Must not have been estimated for humans. 
Based on these inclusion criteria, we ended up with 181 estimated $\mathbf{G}$ matrices (Fig S2). For each published $\mathbf{G}$ matrix, we calculated $\lambda_{2} / \lambda_{1}$ using a purpose-built R Shiny App (ink). unique study ID), taxonomic information (Kingdom through species epithet), trait category

420 (life-history, physiology, morphology, behavior or mixed), the number of traits in the 421 matrix, $\lambda_{1}, \lambda_{2}, \lambda_{2} / \lambda_{1}$, number of dimensions ${ }^{40}$, number of dimensions divided by the 422 number of traits, and all bibliographic information.

Phylogenetic Signal in $\lambda_{2} / \lambda_{1}$

424 To test for phylogenetic signal we fit a simple taxonomic mixed-effects model. This

425 modeling approach incorporates the hierarchical non-independence due to taxonomic 426 relationships but does not require a full phylogeny ${ }^{41}$. Essentially, at each node of a 427 phylogeny, relationships are modeled according to a star relationship. Each taxonomic 428 grouping was included as a random effect, as was study ID, and the resulting model fit with

429 the lme 4 package in $\mathrm{R}^{42}$. From this model we estimated phylogenetic signal as the proportion of variation attributable to taxonomy, the variation attributable to study ID, and

431 the residual variance. Confidence intervals were then estimated based on likelihood profile 432 likelihoods.

433 Comparison of Observed Results to Simulation Results

434 Finally, we compared the observed values to the average for each of the simulation using 435 the intercept coefficient of the above linear model. For this, $t$ was calculated as 43 :

$$
t=\frac{\hat{\beta}-\beta_{H_{0}}}{\text { s.e. }(\hat{\beta})}
$$

437 where $\hat{\beta}$ was the estimated intercept from the taxonomic model (above) and $\beta_{H_{0}}$ was a

438 simulation average. $\mathrm{p}$ was calculated with degrees of freedom estimated using

439 Satterthwaite's method $(\mathrm{df}=17.275)$.

\section{Supplemental Results}

\section{$441 \quad$ Simulation Models}




\section{Statistical Comparison of Evolutionary Metrics}

443 Populations that evolved on different landscapes (drift alone, Gaussian, or holey)

444 significantly differed from each other in the structure of $\mathbf{G}$ after 100 generations (Tables S3

445 - S7). Holey landscapes were characterized by a compression of most variation into the

446 dominant dimension in multivariate space (Tables S3 \& S4; Figures 2 \& S3). Populations

447 evolving on Gaussian landscapes were characterized by a drastic reduction in the total

448 variation present, which was also reflected in reduced evolvability (Tables S5 \& S6; Figures

449 S4 \& S5). The combination of high standing genetic variation and this variation being

450 distributed across dimensions led to populations that evolved solely due to drift to exhibit

451 significantly greater autonomy than observed in any of the other modeling scenarios (Table

452 S7; Figure S6). This greater constraint in populations evolving on either Gaussian or holey

453 landscapes is likely due to the loss of variation for populations evolving on Gaussian

454 landscapes (Figures S4 \& S5) and the compression of variation for populations evolving on

455 holey landscapes (Figures 2 \& S3). 
457 Table S3. ANOVA and Tukey HSD results for $\lambda_{2} / \lambda_{1}$. Significantly greater genetic variation 458 was maintained across all dimensions when populations evolved on Gaussian landscapes 459 or due to drift than when evolving on holey landscapes (Figure 2, main text).

\begin{tabular}{|c|c|c|c|c|c|}
\hline \multicolumn{6}{|l|}{ ANOVA Results } \\
\hline & $\mathrm{df}$ & SS & MSS & $\mathrm{F}$ & $\mathrm{p}$ \\
\hline Simulation type & 5 & 54.98 & 10.996 & 343.5 & $<0.01$ \\
\hline Residual & 1494 & 47.82 & 0.032 & & \\
\hline \multicolumn{6}{|l|}{ Tukey HSD } \\
\hline \multicolumn{2}{|c|}{ Simulation Comparison } & Difference & Lower & Upper & $\mathrm{p}$ \\
\hline \multicolumn{2}{|c|}{ Holey $\mathrm{p}=0.5$-Holey $\mathrm{p}=0.2$} & -0.026 & -0.071 & 0.020 & 0.589 \\
\hline \multicolumn{2}{|c|}{ Holey $\mathrm{p}=0.8$-Holey $\mathrm{p}=0.2$} & 0.011 & -0.035 & 0.057 & 0.984 \\
\hline \multicolumn{2}{|c|}{ Wright 0.1 -Holey $\mathrm{p}=0.2$} & 0.293 & 0.248 & 0.339 & $<0.01$ \\
\hline \multicolumn{2}{|c|}{ Wright 0.5 -Holey $\mathrm{p}=0.2$} & 0.374 & 0.329 & 0.420 & $<0.01$ \\
\hline \multicolumn{2}{|c|}{ Drift-Holey $p=0.2$} & 0.437 & 0.391 & 0.483 & $<0.01$ \\
\hline \multicolumn{2}{|c|}{ Holey $\mathrm{p}=0.8$-Holey $\mathrm{p}=0.5$} & 0.037 & -0.009 & 0.082 & 0.198 \\
\hline \multicolumn{2}{|c|}{ Wright 0.1 -Holey $p=0.5$} & 0.319 & 0.273 & 0.365 & $<0.01$ \\
\hline \multicolumn{2}{|c|}{ Wright 0.5 -Holey $\mathrm{p}=0.5$} & 0.400 & 0.354 & 0.446 & $<0.01$ \\
\hline \multicolumn{2}{|c|}{ Drift-Holey $p=0.5$} & 0.463 & 0.417 & 0.508 & $<0.01$ \\
\hline \multicolumn{2}{|c|}{ Wright 0.1 -Holey $\mathrm{p}=0.8$} & 0.282 & 0.237 & 0.328 & $<0.01$ \\
\hline \multicolumn{2}{|c|}{ Wright 0.5 -Holey $\mathrm{p}=0.8$} & 0.363 & 0.318 & 0.409 & $<0.01$ \\
\hline \multicolumn{2}{|c|}{ Drift-Holey $\mathrm{p}=0.8$} & 0.426 & 0.380 & 0.472 & $<0.01$ \\
\hline \multicolumn{2}{|c|}{ Wright 0.5 -Wright 0.1} & 0.081 & 0.035 & 0.127 & $<0.01$ \\
\hline \multicolumn{2}{|c|}{ Drift-Wright 0.1} & 0.144 & 0.098 & 0.189 & $<0.01$ \\
\hline \multicolumn{2}{|l|}{ Drift-Wright 0.5} & 0.063 & 0.017 & 0.108 & $<0.01$ \\
\hline
\end{tabular}

461 Table S4. ANOVA and Tukey HSD results for $\lambda_{1} / \sum \lambda$. Significantly greater proportional 462 genetic variation was retained in the dominant multivariate direction for populations that 463 evolved on Gaussian landscapes or via drift than when evolving on holey landscapes $464 \quad$ (Figure S3).

\begin{tabular}{|c|c|c|c|c|c|}
\hline \multicolumn{6}{|l|}{ ANOVA Results } \\
\hline & $\mathrm{df}$ & SS & MSS & $\mathrm{F}$ & $\mathrm{p}$ \\
\hline Simulation type & 5 & 29.49 & 5.90 & 325.4 & $<0.01$ \\
\hline Residual & 1494 & 27.08 & 0.02 & & \\
\hline \multicolumn{6}{|l|}{ Tukey HSD } \\
\hline \multicolumn{2}{|c|}{ Simulation Comparison } & Difference & Lower & Upper & $\mathrm{p}$ \\
\hline \multicolumn{2}{|c|}{ Holey $\mathrm{p}=0.5$-Holey $\mathrm{p}=0.2$} & 0.044 & 0.010 & 0.079 & $<0.01$ \\
\hline \multicolumn{2}{|c|}{ Holey $\mathrm{p}=0.8$-Holey $\mathrm{p}=0.2$} & 0.019 & -0.015 & 0.054 & 0.594 \\
\hline \multicolumn{2}{|c|}{ Wright 0.1 -Holey $\mathrm{p}=0.2$} & -0.188 & -0.223 & -0.154 & $<0.01$ \\
\hline \multicolumn{2}{|c|}{ Wright 0.5 -Holey $\mathrm{p}=0.2$} & -0.233 & -0.268 & -0.199 & $<0.01$ \\
\hline \multicolumn{2}{|c|}{ Drift-Holey $p=0.2$} & -0.320 & -0.354 & -0.285 & $<0.01$ \\
\hline \multicolumn{2}{|c|}{ Holey $\mathrm{p}=0.8$-Holey $\mathrm{p}=0.5$} & -0.025 & -0.059 & 0.009 & 0.307 \\
\hline \multicolumn{2}{|c|}{ Wright 0.1 -Holey $p=0.5$} & -0.232 & -0.267 & -0.198 & $<0.01$ \\
\hline \multicolumn{2}{|c|}{ Wright 0.5 -Holey $\mathrm{p}=0.5$} & -0.278 & -0.312 & -0.243 & $<0.01$ \\
\hline \multicolumn{2}{|c|}{ Drift-Holey $p=0.5$} & -0.364 & -0.398 & -0.330 & $<0.01$ \\
\hline \multicolumn{2}{|c|}{ Wright 0.1 -Holey $\mathrm{p}=0.8$} & -0.208 & -0.242 & -0.173 & $<0.01$ \\
\hline \multicolumn{2}{|c|}{ Wright 0.5 -Holey $\mathrm{p}=0.8$} & -0.253 & -0.287 & -0.218 & $<0.01$ \\
\hline \multicolumn{2}{|c|}{ Drift-Holey $p=0.8$} & -0.339 & -0.374 & -0.305 & $<0.01$ \\
\hline \multicolumn{2}{|c|}{ Wright 0.5 -Wright 0.1} & -0.045 & -0.080 & -0.011 & $<0.01$ \\
\hline \multicolumn{2}{|c|}{ Drift-Wright 0.1} & -0.132 & -0.166 & -0.097 & $<0.01$ \\
\hline \multicolumn{2}{|l|}{ Drift-Wright 0.5} & -0.086 & -0.121 & -0.052 & $<0.01$ \\
\hline
\end{tabular}


465 Table S5. ANOVA and Tukey HSD results for the total genetic variation in populations at the 466 end of simulations $\sum \lambda$. The amount of total variation significantly varied across simulation 467 types. Populations that evolved on Gaussian landscapes lost considerably more genetic 468 variation than those evolving on other landscapes (Figure S4).

\begin{tabular}{|c|c|c|c|c|c|}
\hline \multicolumn{6}{|l|}{ ANOVA Results } \\
\hline & $\mathrm{df}$ & SS & MSS & $\mathrm{F}$ & $\mathrm{p}$ \\
\hline Simulation type & 5 & 357826 & 71565 & 6.23 & $<0.01$ \\
\hline Residual & 1494 & 17167085 & 11491 & & \\
\hline \multicolumn{6}{|l|}{ Tukey HSD } \\
\hline \multicolumn{2}{|c|}{ Simulation Comparison } & Difference & Lower & Upper & $\mathrm{p}$ \\
\hline \multicolumn{2}{|c|}{ Holey $p=0.5$-Holey $p=0.2$} & -1.050 & -28.408 & 26.308 & 1.000 \\
\hline \multicolumn{2}{|c|}{ Holey $p=0.8$-Holey $p=0.2$} & -18.153 & -45.511 & 9.205 & 0.407 \\
\hline \multicolumn{2}{|c|}{ Wright 0.1 -Holey $p=0.2$} & -37.651 & -65.009 & -10.293 & $<0.01$ \\
\hline \multicolumn{2}{|c|}{ Wright 0.5 -Holey $\mathrm{p}=0.2$} & -37.237 & -64.595 & -9.879 & $<0.01$ \\
\hline \multicolumn{2}{|c|}{ Drift-Holey $p=0.2$} & -27.791 & -55.149 & -0.433 & 0.044 \\
\hline \multicolumn{2}{|c|}{ Holey $p=0.8$-Holey $p=0.5$} & -17.103 & -44.461 & 10.255 & 0.477 \\
\hline \multicolumn{2}{|c|}{ Wright 0.1 -Holey $\mathrm{p}=0.5$} & -36.601 & -63.959 & -9.243 & $<0.01$ \\
\hline \multicolumn{2}{|c|}{ Wright 0.5 -Holey $\mathrm{p}=0.5$} & -36.187 & -63.545 & -8.830 & $<0.01$ \\
\hline \multicolumn{2}{|c|}{ Drift-Holey $p=0.5$} & -26.741 & -54.099 & 0.617 & 0.060 \\
\hline \multicolumn{2}{|c|}{ Wright 0.1 -Holey $\mathrm{p}=0.8$} & -19.498 & -46.856 & 7.860 & 0.324 \\
\hline \multicolumn{2}{|c|}{ Wright 0.5 -Holey $\mathrm{p}=0.8$} & -19.084 & -46.442 & 8.274 & 0.348 \\
\hline \multicolumn{2}{|c|}{ Drift-Holey $\mathrm{p}=0.8$} & -9.638 & -36.996 & 17.720 & 0.916 \\
\hline \multicolumn{2}{|c|}{ Wright 0.5 -Wright 0.1} & 0.414 & -26.944 & 27.771 & 1.000 \\
\hline \multicolumn{2}{|c|}{ Drift-Wright 0.1} & 9.860 & -17.498 & 37.218 & 0.908 \\
\hline \multicolumn{2}{|l|}{ Drift-Wright 0.5} & 9.446 & -17.912 & 36.804 & 0.923 \\
\hline
\end{tabular}

469 Table S6. ANOVA and Tukey HSD results for evolvability, ē. Because more genetic variation 470 was maintained when populations evolved on holey landscapes or drift (Table S5), 471 evolvability was significantly lower when populations evolved on Gaussian landscapes 472 (Figure S5). (evolvability is just the matrix trace divided by the number of traits)

\begin{tabular}{|c|c|c|c|c|c|}
\hline \multicolumn{6}{|l|}{ ANOVA Results } \\
\hline & $\mathrm{df}$ & SS & MSS & $\mathrm{F}$ & $\mathrm{p}$ \\
\hline Simulation type & 5 & 3578 & 715.7 & 6.23 & $<0.01$ \\
\hline Residual & 1494 & 171671 & 114.9 & & \\
\hline \multicolumn{6}{|l|}{ Tukey HSD } \\
\hline \multicolumn{2}{|c|}{ Simulation Comparison } & Difference & Lower & Upper & $\mathrm{p}$ \\
\hline \multirow{2}{*}{\multicolumn{2}{|c|}{$\begin{array}{l}\text { Holey } \mathrm{p}=0.5-\text { Holey } \mathrm{p}=0.2 \\
\text { Holev } \mathrm{p}=0.8 \text {-Holev } \mathrm{p}=0.2\end{array}$}} & -0.105 & -2.841 & 2.631 & 1.000 \\
\hline & & -1.815 & -4.551 & 0.921 & 0.407 \\
\hline \multicolumn{2}{|c|}{ Wright $01-$ Holev $n=02$} & -3.765 & -6.501 & -1.029 & $<0.01$ \\
\hline \multicolumn{2}{|c|}{ Wright 0.5 -Holey $p=0.2$} & -3.724 & -6.460 & -0.988 & $<0.01$ \\
\hline \multicolumn{2}{|c|}{ Drift-Holey $p=0.2$} & -2.779 & -5.515 & -0.043 & 0.044 \\
\hline \multicolumn{2}{|c|}{ Holey $p=0.8$-Holey $p=0.5$} & -1.710 & -4.446 & 1.025 & 0.477 \\
\hline \multicolumn{2}{|c|}{ Wright 0.1 -Holey $p=0.5$} & -3.660 & -6.396 & -0.924 & $<0.01$ \\
\hline \multicolumn{2}{|c|}{ Wright 0.5 -Holey $p=0.5$} & -3.619 & -6.355 & -0.883 & $<0.01$ \\
\hline \multicolumn{2}{|c|}{ Drift-Holey $p=0.5$} & -2.674 & -5.410 & 0.062 & 0.060 \\
\hline \multicolumn{2}{|c|}{ Wright 0.1 -Holey $\mathrm{p}=0.8$} & -1.950 & -4.686 & 0.786 & 0.324 \\
\hline \multicolumn{2}{|c|}{ Wright 0.5 -Holey $\mathrm{p}=0.8$} & -1.908 & -4.644 & 0.827 & 0.348 \\
\hline \multicolumn{2}{|c|}{ Drift-Holey $p=0.8$} & -0.964 & -3.700 & 1.772 & 0.916 \\
\hline \multicolumn{2}{|c|}{ Wright 0.5 -Wright 0.1} & 0.041 & -2.694 & 2.777 & 1.000 \\
\hline \multicolumn{2}{|c|}{ Drift-Wright 0.1} & 0.986 & -1.750 & 3.722 & 0.908 \\
\hline \multicolumn{2}{|l|}{ Drift-Wright 0.5} & 0.945 & -1.791 & 3.680 & 0.923 \\
\hline
\end{tabular}


473 Table S7. ANOVA and Tukey HSD results for autonomy, ā. Significantly greater variation 474 was maintained across all dimensions when populations evolved on Gaussian landscapes

475 or due to drift than when evolving on holey landscapes (Figure S6).

\begin{tabular}{|c|c|c|c|c|c|}
\hline \multicolumn{6}{|l|}{ ANOVA Results } \\
\hline & $\mathrm{df}$ & SS & MSS & $\mathrm{F}$ & $\mathrm{p}$ \\
\hline Simulation type & 5 & 43.61 & 8.72 & 518.3 & $<0.01$ \\
\hline Residual & 1494 & 25.14 & 0.02 & & \\
\hline \multicolumn{6}{|l|}{ Tukey HSD } \\
\hline \multicolumn{2}{|c|}{ Simulation Comparison } & Difference & Lower & Upper & $\bar{p}$ \\
\hline \multicolumn{2}{|c|}{ Holey $\mathrm{p}=0.5$-Holey $\mathrm{p}=0.2$} & -0.021 & -0.054 & 0.012 & 0.479 \\
\hline \multicolumn{2}{|c|}{ Holey $\mathrm{p}=0.8$-Holey $\mathrm{p}=0.2$} & -0.042 & -0.075 & -0.009 & $<0.01$ \\
\hline \multicolumn{2}{|c|}{ Wright 0.1 -Holey $\mathrm{p}=0.2$} & -0.148 & -0.181 & -0.115 & $<0.01$ \\
\hline \multicolumn{2}{|c|}{ Wright 0.5 -Holey $\mathrm{p}=0.2$} & 0.395 & 0.362 & 0.428 & $<0.01$ \\
\hline \multicolumn{2}{|c|}{ Drift-Holey $p=0.2$} & 0.077 & 0.044 & 0.110 & $<0.01$ \\
\hline \multicolumn{2}{|c|}{ Holey $\mathrm{p}=0.8$-Holey $\mathrm{p}=0.5$} & -0.022 & -0.055 & 0.011 & 0.424 \\
\hline \multicolumn{2}{|c|}{ Wright 0.1 -Holey $p=0.5$} & -0.127 & -0.160 & -0.094 & $<0.01$ \\
\hline \multicolumn{2}{|c|}{ Wright 0.5 -Holey $\mathrm{p}=0.5$} & 0.415 & 0.382 & 0.448 & $<0.01$ \\
\hline \multicolumn{2}{|c|}{ Drift-Holey $p=0.5$} & 0.098 & 0.064 & 0.131 & $<0.01$ \\
\hline \multicolumn{2}{|c|}{ Wright 0.1 -Holey $\mathrm{p}=0.8$} & -0.106 & -0.139 & -0.072 & $<0.01$ \\
\hline \multicolumn{2}{|c|}{ Wright 0.5 -Holey $\mathrm{p}=0.8$} & 0.437 & 0.404 & 0.470 & $<0.01$ \\
\hline \multicolumn{2}{|c|}{ Drift-Holey $\mathrm{p}=0.8$} & 0.119 & 0.086 & 0.152 & $<0.01$ \\
\hline \multicolumn{2}{|c|}{ Wright 0.5 -Wright 0.1} & 0.543 & 0.509 & 0.576 & $<0.01$ \\
\hline \multicolumn{2}{|c|}{ Drift-Wright 0.1} & 0.225 & 0.192 & 0.258 & $<0.01$ \\
\hline \multicolumn{2}{|l|}{ Drift-Wright 0.5} & -0.318 & -0.351 & -0.285 & $<0.01$ \\
\hline
\end{tabular}

477 For populations evolving on Gaussian landscapes, compression of genetic variation into the

478 leading dimension decreased with increasing heritability and an increasing strength of

479 selection (Table S8, Figure S7). No two-way interaction was statistically significant. Put

480 another way, $\lambda_{2} / \lambda_{1}$, increased with heritability and the strength of selection and average

$481 \lambda_{2} / \lambda_{1}$ was 0.68 for average parameter values (Table $S 8$ ).

482 For populations evolving solely due to drift, $\lambda_{2} / \lambda_{1}$ increased with greater initial

483 total genetic variation (Table S9). However, the strength of this effect was minimal. More

484 dramatically, $\lambda_{2} / \lambda_{1}$ significantly and strongly decreased with increasing average initial

485 absolute genetic correlation (Table $S 9$ ). At the extreme, $\lambda_{2} / \lambda_{1}$ approached 0 as the average

486 initial absolute correlation approaches 1 . No two-way interaction was statistically

487 significant. Average $\lambda_{2} / \lambda_{1}$ was 0.69 for average parameter values (Table S9). 
When evolving on holey landscapes, and consistent with prior simulation

490 Compression into a single dimension also increased with increasing heritability and

491 increasing average absolute initial correlations (Table S10).

494 selection on Gaussian surfaces (Tables S8 - S10; Figures S7 - S9). This was a surprisingly

495 robust result regardless of the starting parameters of a simulation (Figures S7 - S9). This

496 parameter robustness ${ }^{44}$ supports the generality of our modeling. Unfortunately, we were

497 not able to investigate other forms of robustness ${ }^{44}$ due to computational limitations.

498 Table S8. Linear modeling results for Gaussian landscape parameter exploration. All 499 covariates were modeled while centered (but not variance standardized).

\begin{tabular}{lllll}
\hline Covariate & Estimate & $\begin{array}{l}\text { Standard } \\
\text { Error }\end{array}$ & $\mathrm{t}^{*}$ & $\mathrm{p}$ \\
\hline Intercept (average) & 0.680 & 0.004 & 157.94 & $<0.01$ \\
Total variation (tot. var) & 0.004 & 0.003 & 1.33 & 0.182 \\
Mean |correlation| (mean cor) & -0.256 & 0.170 & -1.51 & 0.132 \\
h $^{2}$ & $\mathbf{0 . 1 0 3}$ & $\mathbf{0 . 0 1 5}$ & $\mathbf{6 . 7 0}$ & $<\mathbf{0 . 0 1}$ \\
Selection strength (ss) & $\mathbf{0 . 0 6 9}$ & $\mathbf{0 . 0 1 9}$ & $\mathbf{3 . 6 0}$ & $<\mathbf{0 . 0 1}$ \\
tot.var $\times$ mean cor & -0.070 & 0.107 & -0.66 & 0.513 \\
tot.var $\times \mathrm{h}^{2}$ & -0.008 & 0.010 & -0.75 & 0.454 \\
tot.var $\times$ ss & 0.012 & 0.013 & 0.95 & 0.344 \\
mean cor $\times \mathrm{h}^{2}$ & 0.806 & 0.601 & 1.34 & 0.180 \\
mean cor $\times$ ss & 0.384 & 0.733 & 0.52 & 0.600 \\
$\mathrm{~h}^{2} \times$ ss & -0.098 & 0.070 & -1.39 & 0.164 \\
\hline
\end{tabular}

${ }^{*} \mathrm{p}$ values are based on this $\mathrm{t}$ value with 989 degrees of freedom

500 Table S9. Linear modeling results for parameter exploration of the drift model. All 501 covariates were modeled while centered (but not variance standardized).

\begin{tabular}{lllll}
\hline Covariate & Estimate & $\begin{array}{l}\text { Standard } \\
\text { Error }\end{array}$ & $\mathrm{t}^{*}$ & $\mathrm{p}$ \\
\hline Intercept (average) & 0.689 & 0.004 & 165.12 & $<0.01$ \\
Total variation (tot. var) & $\mathbf{0 . 0 0 9}$ & $\mathbf{0 . 0 0 3}$ & $\mathbf{3 . 6 2}$ & $<\mathbf{0 . 0 1}$ \\
Mean |correlation| (mean cor) & $\mathbf{- 0 . 8 6 7}$ & $\mathbf{0 . 1 6 0}$ & $\mathbf{- 5 . 4 1}$ & $<\mathbf{0 . 0 1}$ \\
$\mathrm{h}^{2}$ & 0.004 & 0.015 & 0.27 & 0.786 \\
tot.var $\times$ mean cor & -0.034 & 0.103 & -0.33 & 0.740 \\
tot.var $\times \mathrm{h}^{2}$ & 0.015 & 0.009 & 1.63 & 0.103 \\
mean cor $\times \mathrm{h}^{2}$ & -0.209 & 0.555 & -0.38 & 0.706 \\
\hline
\end{tabular}

${ }^{*} \mathrm{p}$ values are based on this t value with 993 degrees of freedom 
502 Table S8. Linear modeling results for holey landscape parameter exploration. All covariates 503 were modeled while centered (but not variance standardized).

\begin{tabular}{lllll}
\hline Covariate & Estimate & $\begin{array}{l}\text { Standard } \\
\text { Error }\end{array}$ & $\mathrm{t}^{*}$ & $\mathrm{p}$ \\
\hline Intercept (average) & 0.423 & 0.007 & 61.03 & $<0.01$ \\
Total variation (tot. var.) & 0.007 & 0.004 & 1.56 & 0.119 \\
Mean |correlation| (mean cor) & -0.195 & 0.265 & -0.74 & 0.462 \\
$\mathbf{h}^{2}$ & $\mathbf{- 0 . 2 7 2}$ & $\mathbf{0 . 0 2 4}$ & $\mathbf{- 1 1 . 2 2}$ & $<\mathbf{0 . 0 1}$ \\
$p$ & 0.011 & 0.024 & 0.47 & 0.640 \\
tot.var $\times$ mean cor & 0.070 & 0.150 & 0.47 & 0.640 \\
tot.var $\times \mathrm{h}^{2}$ & 0.012 & 0.015 & 0.78 & 0.435 \\
tot.var $\times p$ & 0.007 & 0.015 & 0.48 & 0.631 \\
mean cor $\times \mathrm{h}^{2}$ & 0.547 & 0.940 & 0.58 & 0.561 \\
mean cor $\times p$ & -0.239 & 0.941 & -0.26 & 0.799 \\
$\mathbf{h}^{2} \times \boldsymbol{p}$ & $\mathbf{- 0 . 5 9 3}$ & $\mathbf{0 . 0 8 6}$ & $\mathbf{- 6 . 9 3}$ & $<\mathbf{0 . 0 1}$ \\
\hline
\end{tabular}

*p values are based on this $\mathrm{t}$ value with 989 degrees of freedom

$504 \quad$ Empirically Estimated G Matrices

505 Phylogenetic Signal in $\lambda_{2} / \lambda_{1}$

506 Table S9. Variances for $\lambda_{2} / \lambda_{1}$-with associated $95 \%$ confidence intervals - at each

507 taxonomic level, for study ID, and residual. Proportion of variation for taxonomy, study ID, 508 and residual are also provided

\begin{tabular}{lcc}
\hline Variance component & Estimate $(95 \% \mathrm{CI})$ & Proportion of variance \\
\hline Study ID & $0.026(0.013: 0.048)$ & 0.45 \\
\hline Taxonomy & 0.003 & 0.05 \\
species & $0(0: 0.01)$ & \\
Genus & $0(0: 0.016)$ & \\
Family & $0.003(0: 0.02)$ & \\
Order & $0(0: 0.018)$ & \\
Class & $0(0: 0.008)$ & \\
Phylum & $0(0: 0.007)$ & 0.50 \\
Kingdom & $0(0: 0.011)$ & \\
\hline Residual & $0.029(0.023: 0.037)$ &
\end{tabular}

509 Comparison of Observed Results to Simulation Results

510 Observed results did not significantly differ from simulated populations that evolved on

511 holey landscapes (Figure 2; Table S10). 
513 Table S10. $t$ values and associated $p$ values for the comparison of the observed average of $514 \lambda_{2} / \lambda_{1}$ versus the average $\lambda_{2} / \lambda_{1}$ for each set of simulations. The observed average and its 515 standard error was taken from a taxonomic mixed-effects model.

\begin{tabular}{llccc}
\hline $\begin{array}{l}\text { Average } \\
\text { observed } \\
\lambda_{2} / \lambda_{1}\end{array}$ & Simulation & $\begin{array}{l}\text { Simulation } \\
\text { average } \lambda_{2} / \lambda_{1}\end{array}$ & $\mathrm{t}$ & $\mathrm{p}$ \\
& Holey $(\mathrm{p}=0.2)$ & 0.357 & 0.320 & 0.753 \\
& Holey $(\mathrm{p}=0.5)$ & 0.331 & 1.199 & 0.247 \\
& Holey $(\mathrm{p}=0.8)$ & 0.368 & -0.050 & 0.961 \\
$0.366 \mathrm{vs:}$ & $\begin{array}{l}\text { Gaussian } \\
\text { (se: } 0.03)\end{array}$ & 0.650 & -9.66 & $<0.01$ \\
& $\begin{array}{l}\text { Gaussian } \text { prob. }=0.1) \\
\text { (surv. prob. }=0.5)\end{array}$ & 0.731 & -12.416 & $<0.01$ \\
& Drift & 0.794 & -14.552 & $<0.01$ \\
\hline
\end{tabular}

$*$ degrees of freedom $=17.275$ 


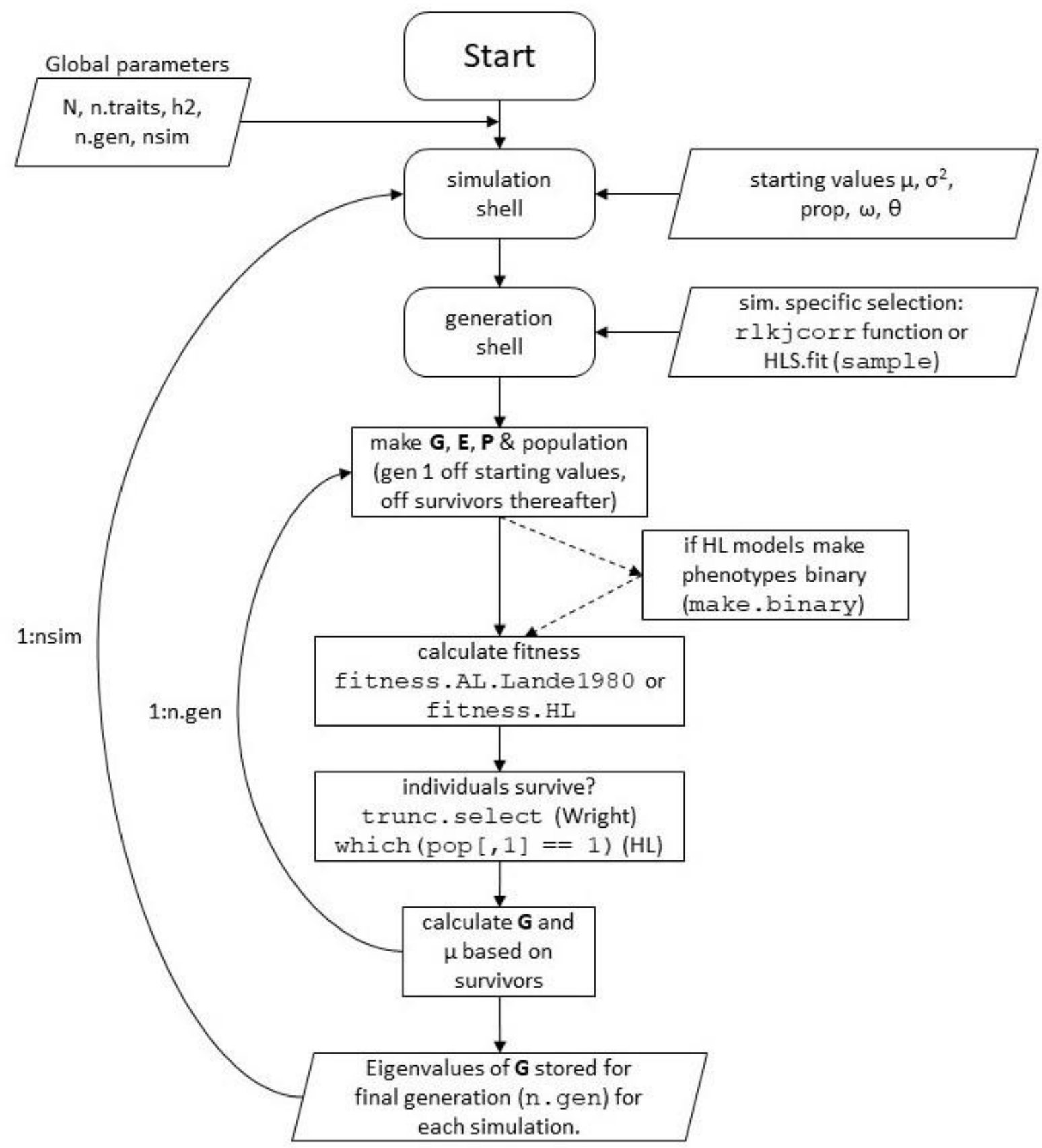

518 Figure S1. Model flow diagram for HL and gaussian landscapes 


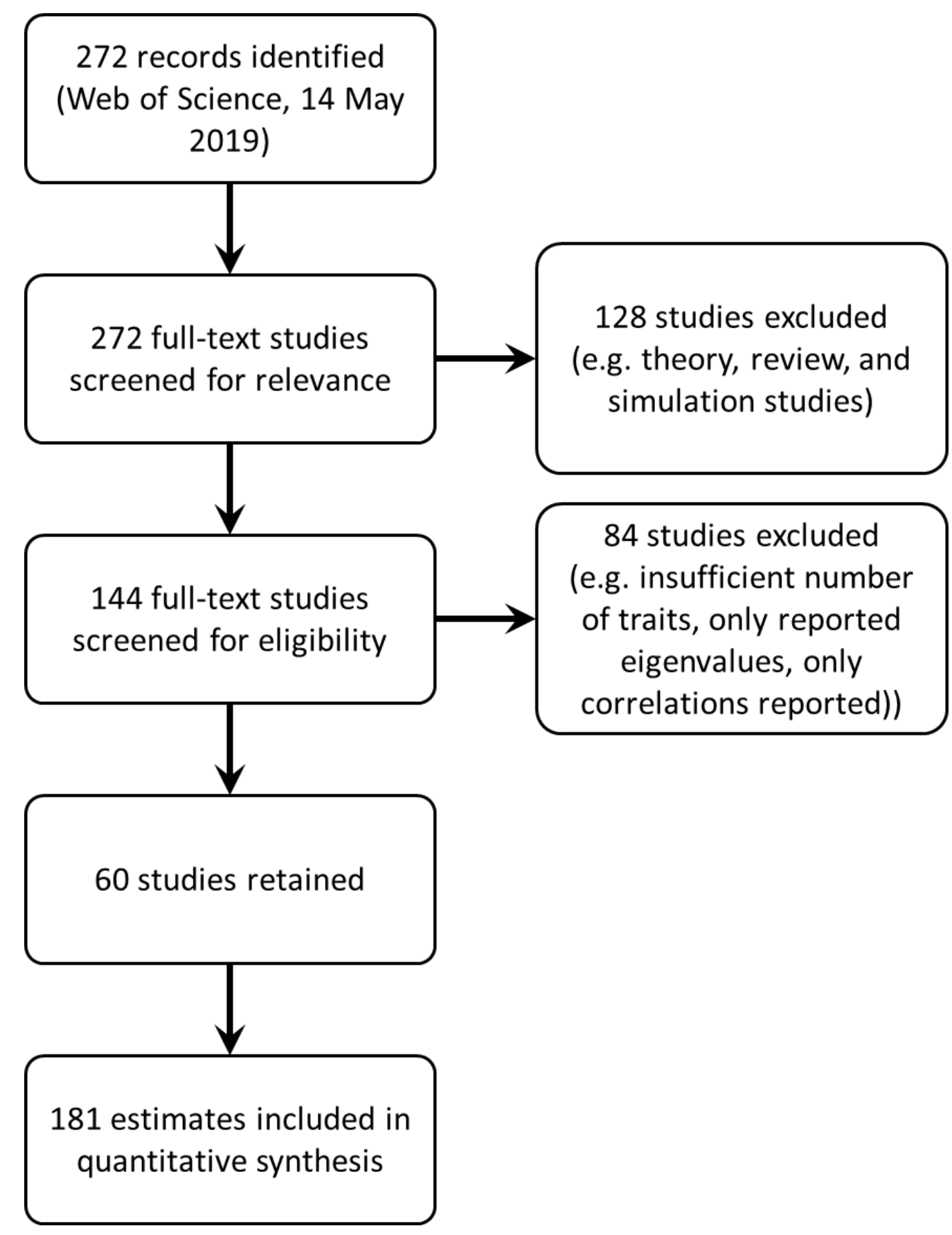

519 Figure S2. PRISMA diagram for studies and estimates included in taxonomic analyses. 


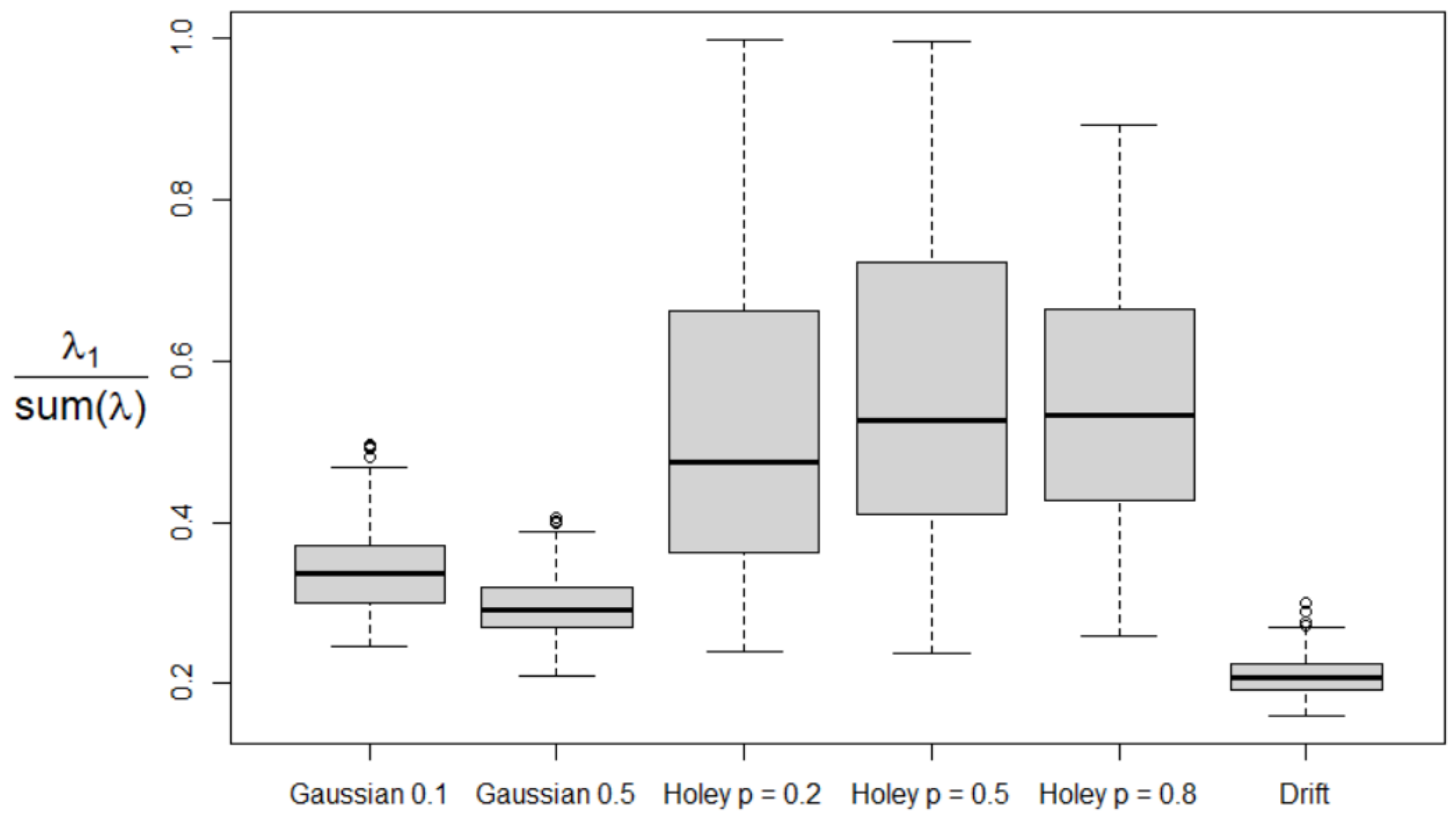

520 Figure S3. Variation was more evenly distributed across dimensions when populations evolved on Gaussian landscapes or due solely to drift. Consequently, less total variation was present in the first dimension (Table S4). 

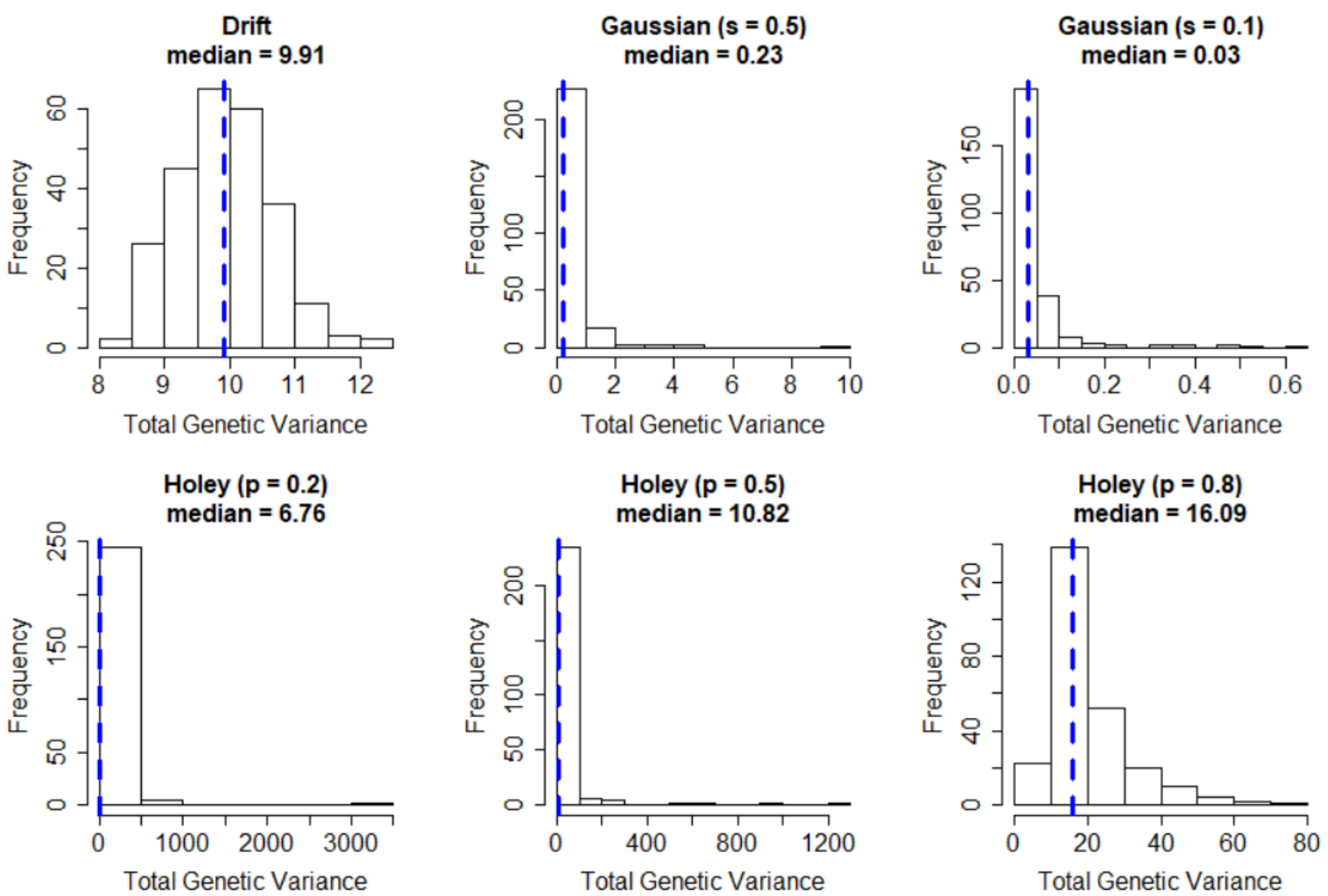

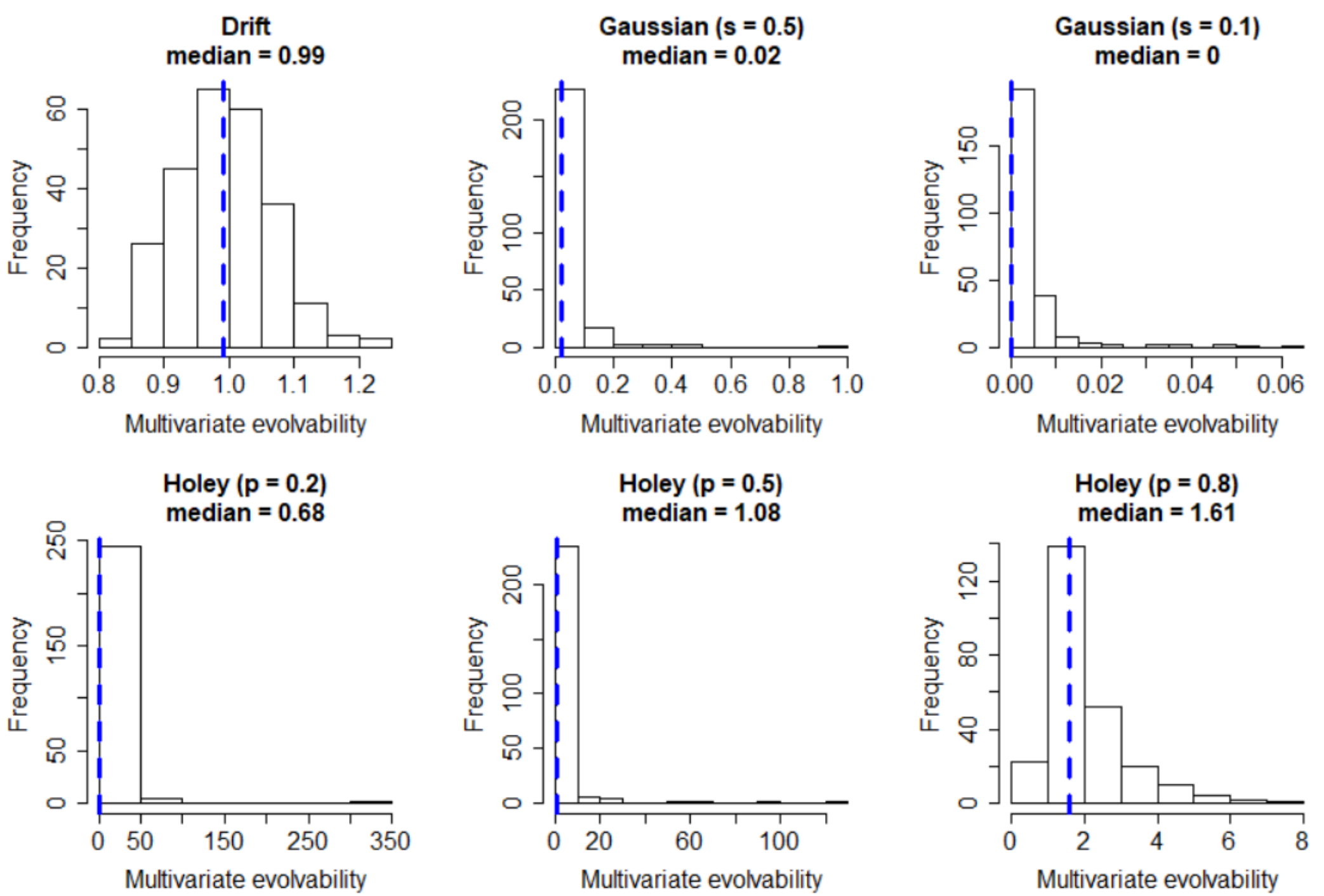

Figure S5. Multivariate evolvability after 100 generations in each of six modeling conditions and across 250 simulations. 

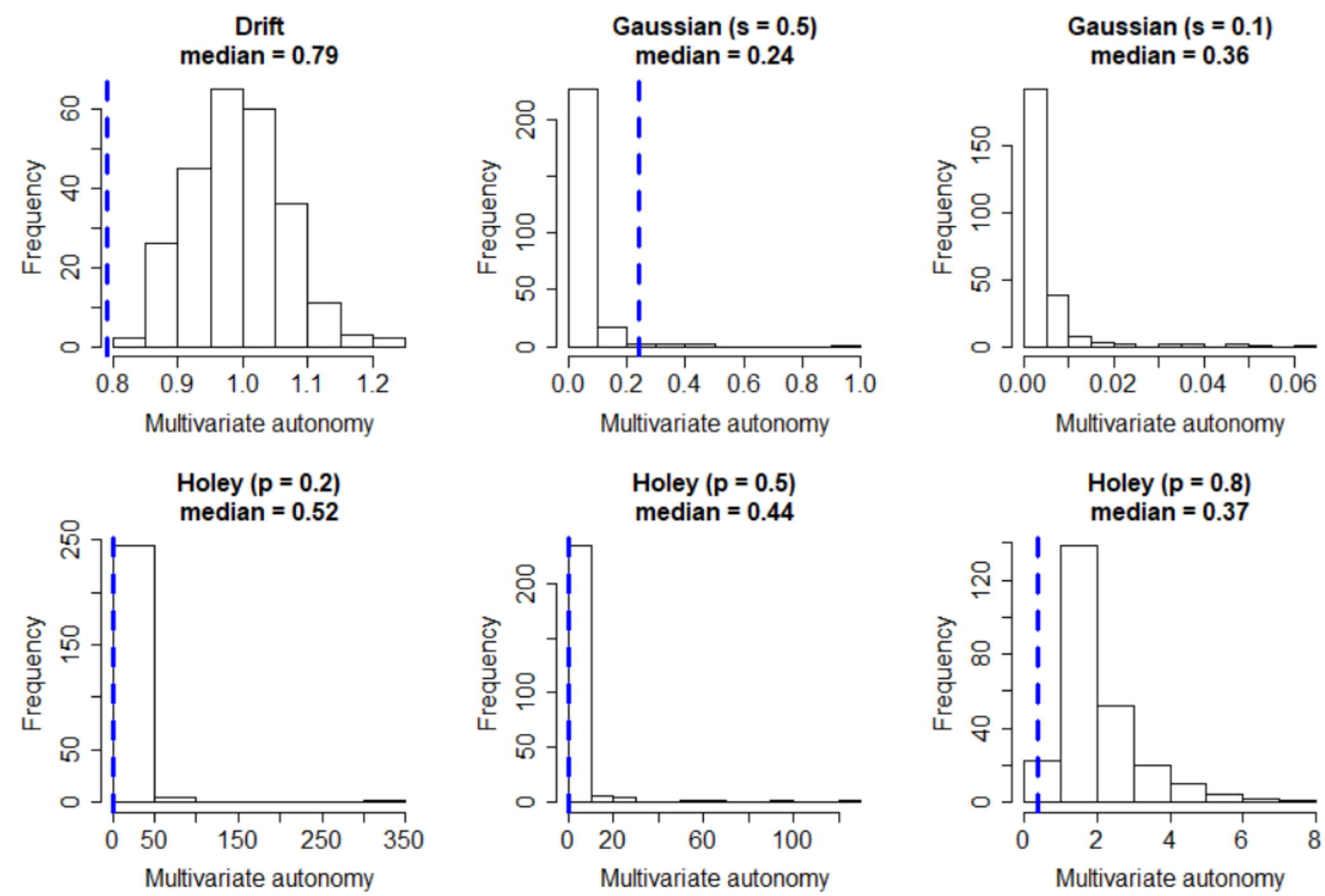

526 Figure S6. Multivariate autonomy after 100 generations in each of six modeling conditions and across 250 simulations.

527 Selection on Gaussian surfaces led to a significant reduction in autonomy (Table S7). 


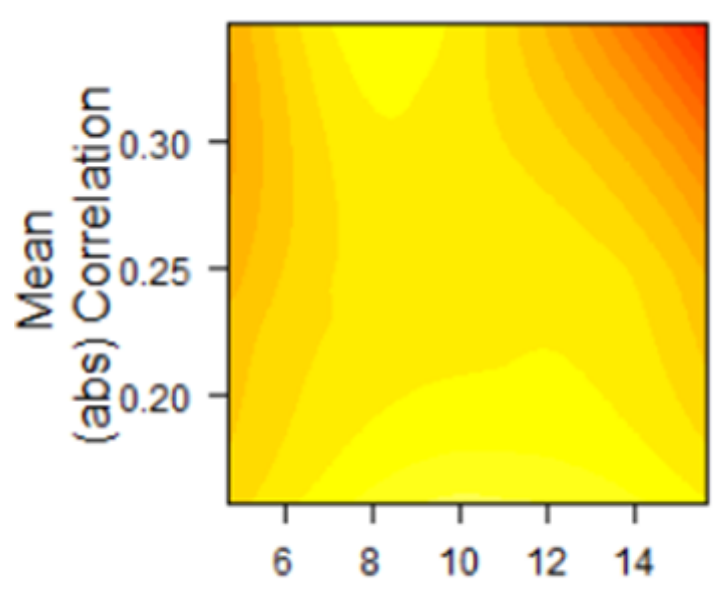

Total Starting Variation

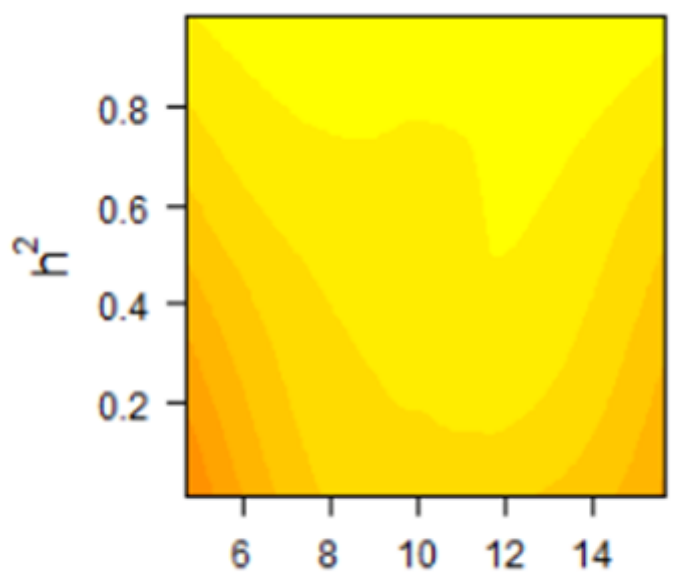

Total Starting Variation

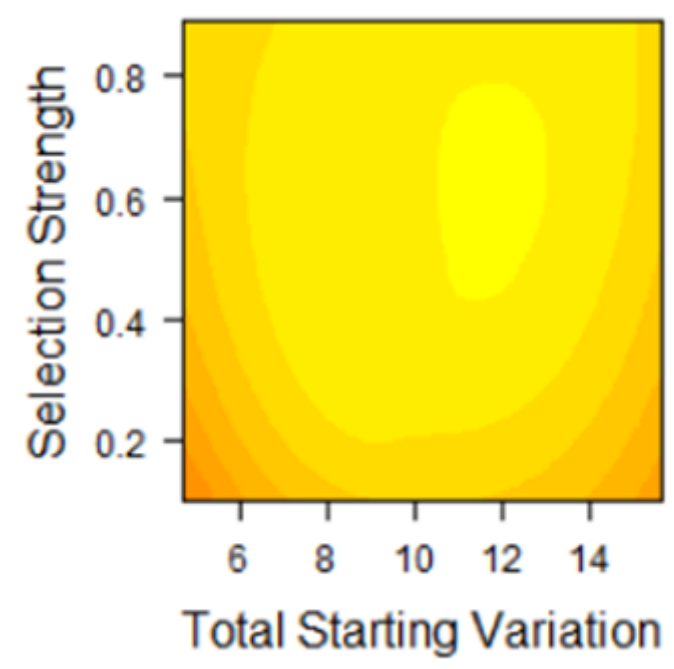

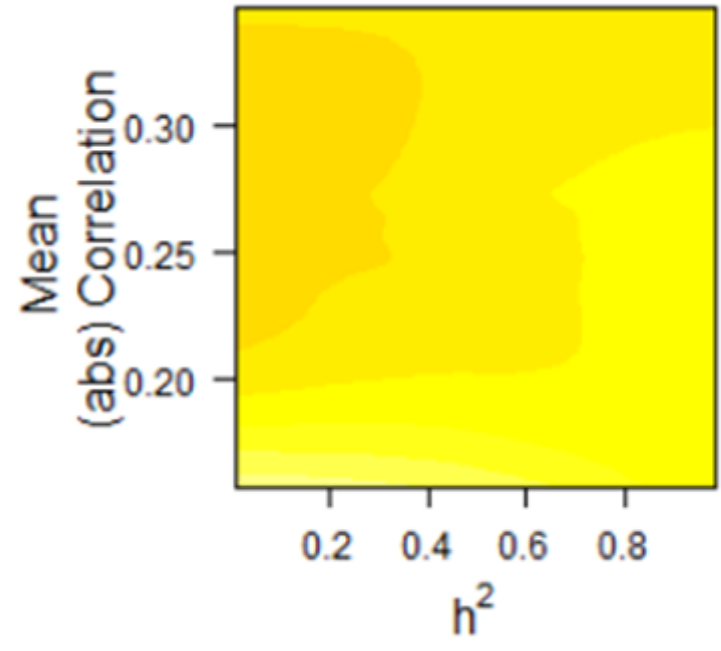

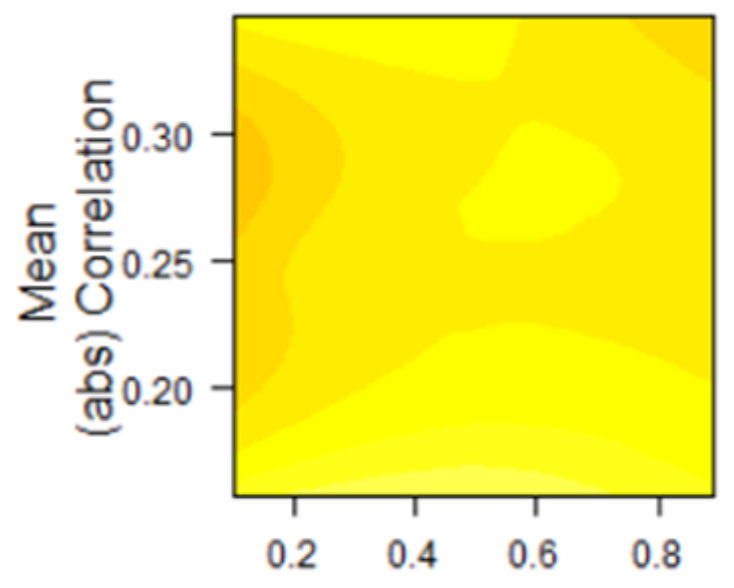

Selection Strength

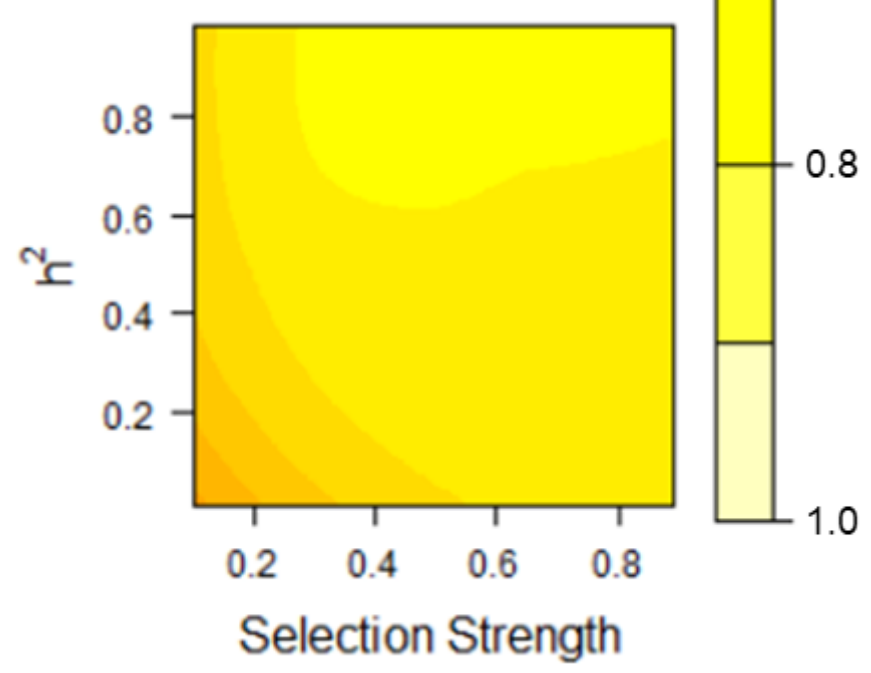

Figure S7. $\lambda_{2} / \lambda_{1}$ after selection on Gaussian surfaces remained high regardless of starting parameters (Table S8). 


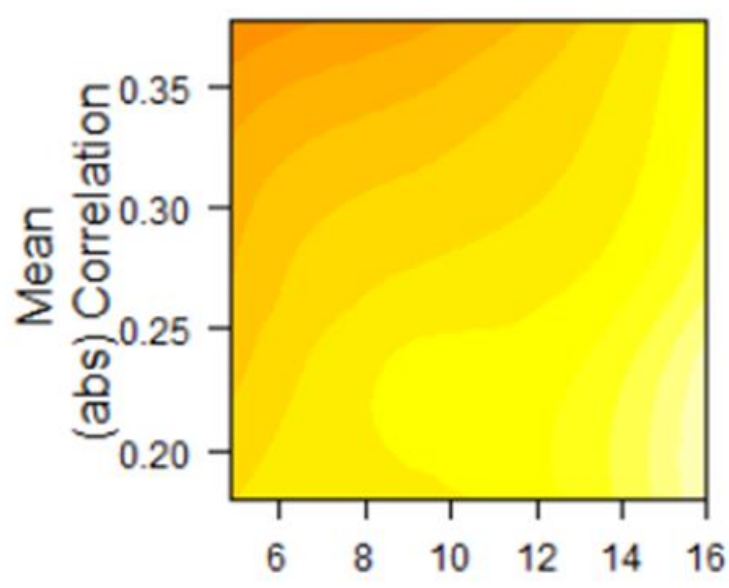

Total Starting Variation
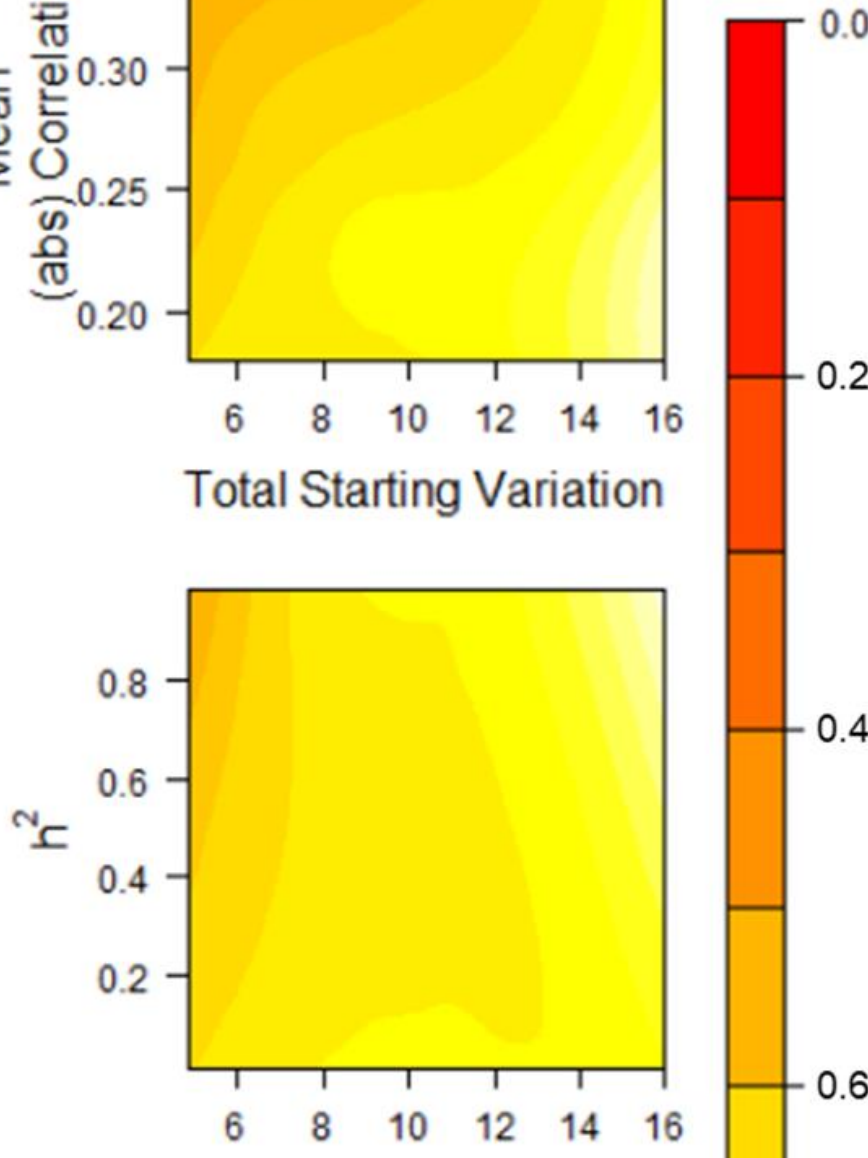

Total Starting Variation

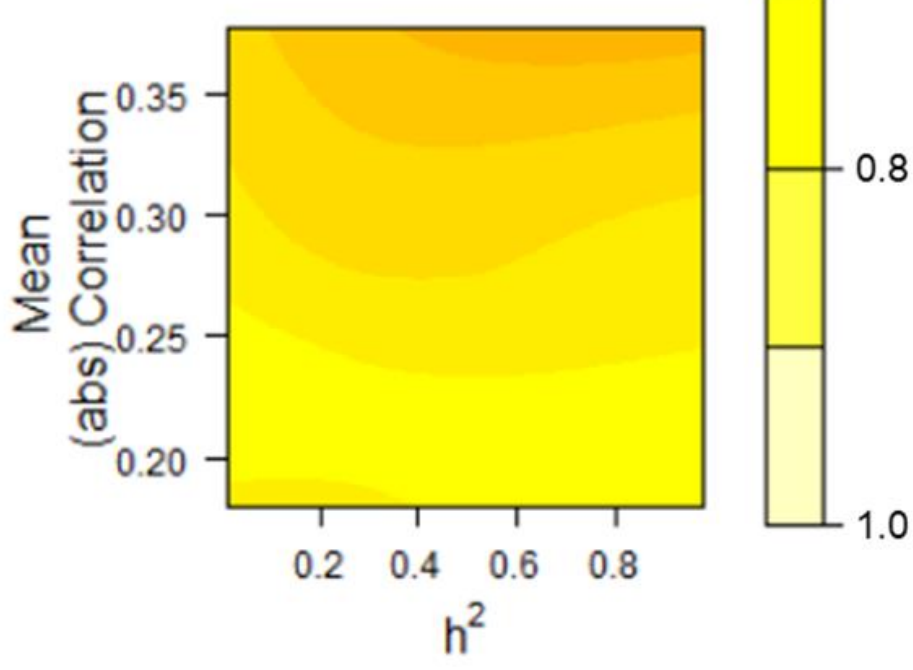

530 Figure S8. $\lambda_{2} / \lambda_{1}$ after evolution due to drift remained high regardless of starting 531 parameters (Table S9). 


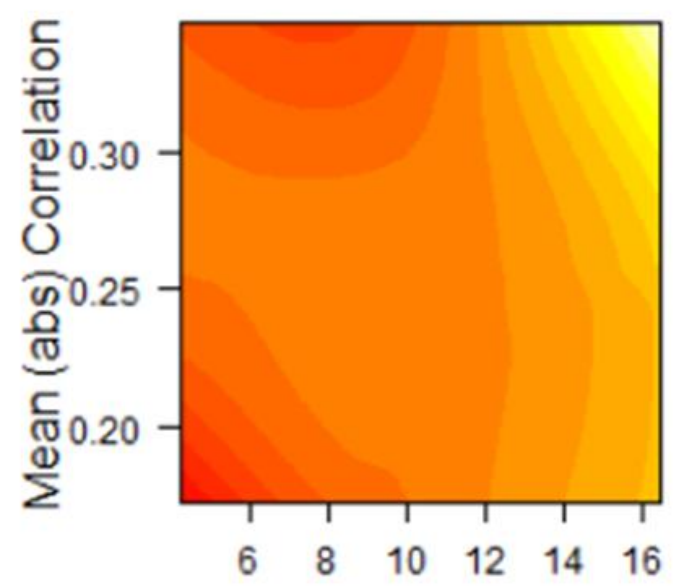

Total Starting Variation
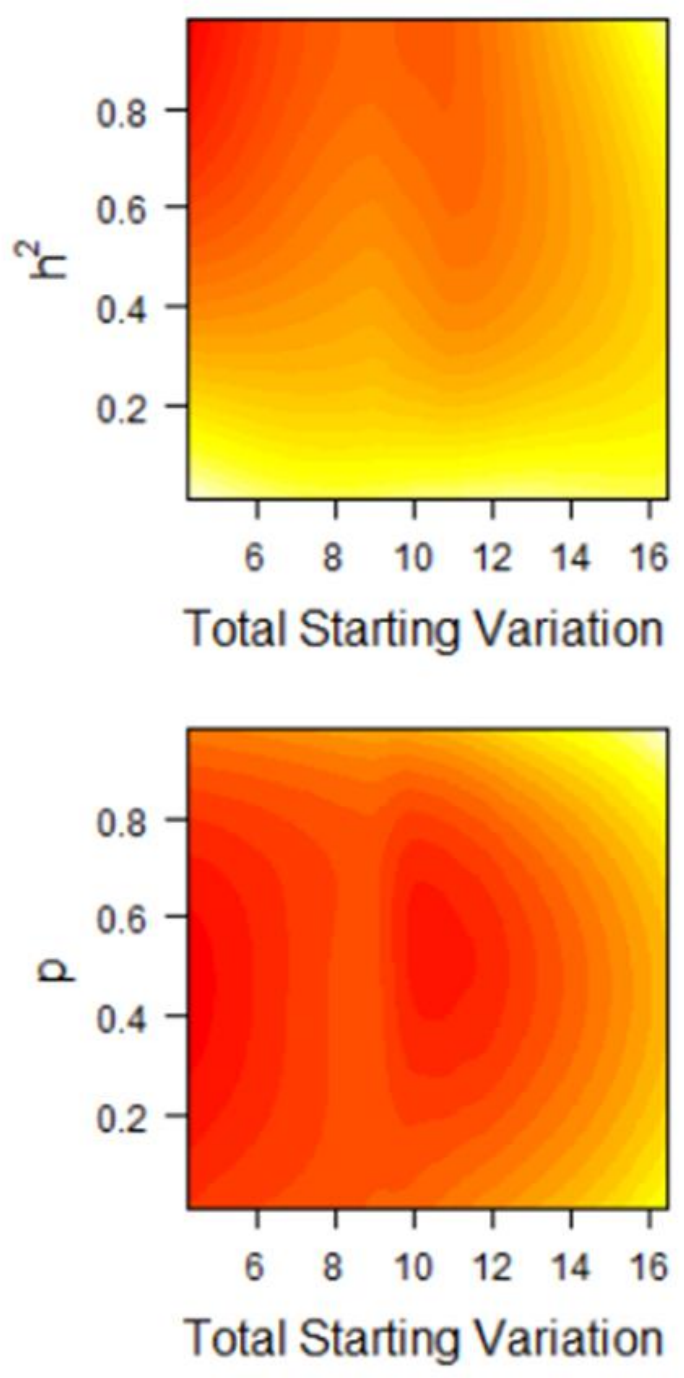
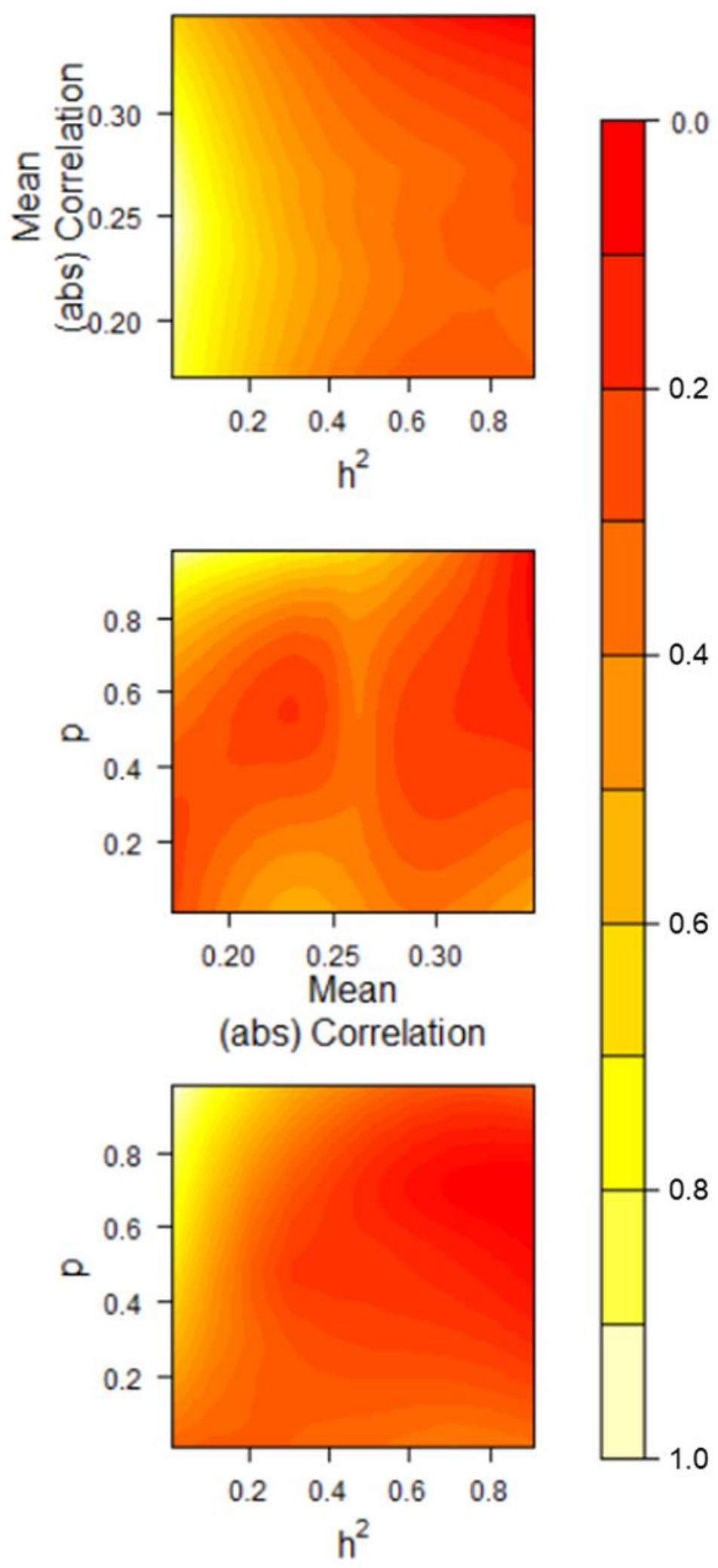

532 Figure $S 9 \lambda_{2} / \lambda_{1}$ after evolution on holey landscapes remained low regardless of starting 533 parameters (Table S10). 